\title{
Geogenic Imprint on Groundwater and Its Quality in Parts of the Mamfe Basin, Manyu Division, Cameroon
}

\author{
Richard Ayuk II Akoachere ${ }^{1^{*}}$ (D), Thomson Areapkoh Eyong1, Sonia Ebot Egbe1, \\ Regina Engome Wotany'1, Michael Obiekwe Nwude'2, Omagbemi Omoloju Yaya ${ }^{2}$ \\ ${ }^{1}$ Department of Geology, University of Buea, Buea, Cameroon \\ ${ }^{2}$ National Water Resources Institute, Kaduna, Nigeria \\ Email: *r.akoachere@ubuea.cm
}

How to cite this paper: Akoachere, R. A. II, Eyong, T. A., Egbe, S. E., Wotany, R. E., Nwude, M. O., \& Yaya, O. O. (2019). Geogenic Imprint on Groundwater and Its Quality in Parts of the Mamfe Basin, Manyu Division, Cameroon. Journal of Geoscience and Environment Protection, 7, 184-211. https://doi.org/10.4236/gep.2019.75016

Received: March 12, 2019

Accepted: May 27, 2019

Published: May 30, 2019

Copyright $\odot 2019$ by author(s) and Scientific Research Publishing Inc. This work is licensed under the Creative Commons Attribution International License (CC BY 4.0).

http://creativecommons.org/licenses/by/4.0/

(c) (i) Open Access

\begin{abstract}
Groundwater studies in parts of the Mamfe basin are sparse and the Mamfe area has the highest population density in the Mamfe basin. An in-depth study of groundwater rock interaction and groundwater quality is of vital importance. This same part of the basin is the economic centre and as such development of businesses in this area requires knowledge of the groundwater quality. Therefore, this study was undertaken to determine the input of the rock formations on the groundwater solute chemistry and groundwater domestic-agro-industrial quality using hydrogeochemical tools and physicochemical parameters: Ionic ratios, Gibbs diagrams, Piper diagrams, Durov diagrams and water quality indices. From physicochemical parameters, in the rainy season, $\mathrm{pH}$ ranged from, $4.3-8.6$; EC, $3-1348 \mu \mathrm{S} / \mathrm{cm}$; Temperature, $24.4^{\circ} \mathrm{C}-30.1^{\circ} \mathrm{C}$ and TDS, $2.01-903.16 \mathrm{mg} / \mathrm{L}$ and in the dry season, $\mathrm{pH}$ ranged from 5.5 - 9.3; EC, 6 - $994 \mu \mathrm{S} / \mathrm{cm}$; Temperature, $25^{\circ} \mathrm{C}-38.6^{\circ} \mathrm{C}$ and TDS, $4.02-632.48 \mathrm{mg} / \mathrm{L}$. Forty groundwater samples: 20 per season, wet and dry were analysed. The major ions fell below WHO acceptable limits for both seasons. The sequences of abundance of major ions were: $\mathrm{Ca}^{2+}>\mathrm{K}^{+}>\mathrm{Mg}^{2+}>$ $\mathrm{NH}_{4}^{+}>\mathrm{Na}^{+}, \mathrm{Cl}^{-}>\mathrm{HCO}_{3}^{-}>\mathrm{SO}_{4}^{2-}>\mathrm{HPO}_{4}^{2-}>\mathrm{NO}_{3}$ in wet season and $\mathrm{Ca}^{2+}>\mathrm{Mg}^{2+}>\mathrm{K}^{+}>\mathrm{Na}^{+}, \mathrm{HCO}_{3}^{-}>\mathrm{Cl}^{-}>\mathrm{SO}_{4}^{2-}>\mathrm{HPO}_{4}^{2-}>\mathrm{NO}_{3}^{-}$in dry season. Ion-exchange, simple dissolution and uncommon dissolution processes determined groundwater character. Groundwater ionic content was as a result of ion exchange from rock-weathering. Water types are: $\mathrm{CaSO}_{4}$ and $\mathrm{MgHCO}_{3}$ in both seasons. Hydrogeochemical facies are Ca- $\mathrm{Mg}-\mathrm{Cl}-\mathrm{SO}_{4}$ and $\mathrm{Ca}-\mathrm{Mg}-\mathrm{HCO}_{3}$. SAR for wet season $0.05-0.06$ and dry season $0.00-0.05, \% \mathrm{Na}$ wet season $3.64-16.59$ and dry season $1.22-10.97$, KR wet season $0.01-0.02$ and $0.00-0.02$ dry season, PI wet season $0.89-68.63$ and dry season $18.75-$
\end{abstract}


73.35, MAR wet season 13.3 - 67.88 and dry season 27.02 - 77.01, WQI wet season $0-70.79$ and $0-276.60$ dry season, RSC wet season -4.59 to -0.33 and dry season $-5.13-0.31$ and groundwater was excellent-good for irrigation purposes. Some physicochemical parameters: $\mathrm{pH}$, EC and TDS exceeded permissible limits.

\section{Keywords}

Geogenic Imprint, Hydrogeochemical Facies, Groundwater Quality, Mamfe, Cameroon

\section{Introduction}

Water is an important natural resource essential for the existence of life and is basic human entity. Water resources are used for various purposes like drinking, agricultural, industrial, household, recreational, and environmental activities. Groundwater is one of the major sources of drinking water all over the world (Bear, 1979). There has been tremendous increase in the demand for fresh water due to growth in population. Since groundwater is a renewable natural resource and a valuable component of the ecosystem, it is vulnerable to natural and human impacts. It is estimated that approximately one-third of the world's population uses groundwater for drinking (Nickson et al., 2005). In most parts of the Mamfe basin, groundwater is the major source of water supply for drinking and agricultural purposes. Few studies on groundwater quality have been carried out in this area, thus the groundwater might present a potential health hazard as such there was a need to determine the domestic-agro-industrial quality. Groundwater quality data give important clues to the geologic history, rock type and indications of groundwater recharge, discharge and storage (Walton, 1970). Variations in groundwater quality in an area are a function of physical and chemical parameters that are greatly influenced by geological formations and anthropogenic properties (Kumar et al., 2011). According to Babiker et al. (2007), the chemistry of groundwater is not only related to the lithology of the area and the residence time the water is in contact with rock material, but also reflects inputs from the atmosphere, from soil and weathering as well as from pollutant sources such as mining, land clearance, saline intrusion, industrial and domestic wastes. Groundwater used for domestic and irrigation purposes can vary greatly in quality depending upon type and quantity of dissolved salts. It contains a wide variety of dissolved inorganic chemical constituents in various concentrations, resulting from chemical and biochemical interactions between water and the geological materials. Dissolved salts should be present in irrigation water in relatively small but significant amounts. They originate from dissolution or weathering of the rocks and soil, including dissolution of lime, gypsum and other slowly dissolved soil minerals. Research on groundwater studies in parts of the Mamfe basin is sparse and Mamfe area has the highest population density in the 
basin. An in-depth study of groundwater rock interaction and groundwater quality is of vital importance. This same part of the basin is the economic centre and as such development of business in the study area requires knowledge on groundwater quality. Therefore, the present study was carried out to determine the contribution of the formations to groundwater chemistry, groundwater quality and its suitability for drinking and agricultural uses in parts of the Mamfe basin.

\section{Geologic Background of the Study Area}

Mamfe and environs is situate between latitude 5.65 - 5.85N and longitude 9.25 9.55E Figure 1. Manyu Divisionis made up of four sub-divisions and occupied by four main ethnic groups: the Anyangs, Kenyangs, Akwayas, and Ejaghams. It has a population of over 200,000 inhabitants (Mamfe Council, 2014). The area is made up of mostly farmers, business people and civil servants from almost every ethnic group in Cameroon and some from other countries. The climate in this division is hot and humid and consists of a rainy and a dry season modified by the deviation of the monsoon and the relief of Mount Cameroon (Ndip et al., 2018). The vegetation is dominantly that of the equatorial rain forest, and the drainage system is principally that of the Cross River whose main source is found in Mount Bambouto. The sources of its main tributaries the Munaya and Badi Rivers are at Mount Rumpi and Nda Ali respectively (Ndip et al., 2018).

The study area forms part of the Mamfe basin which lies along the Cameroon Volcanic Line. Sedimentation began in the Mamfe basin during the Albian (Dumort, 1968) and lithologies making-up the body of sediments are: basal conglomerates, conglomeratic sandstones, mudstones, shales, calcareous and carbonaceous rocks Figure 2 that are highly fractured (Lordon et al., 2017). The Mamfe basin is a south-eastern trending Cretaceous rift basin that bifurcates off

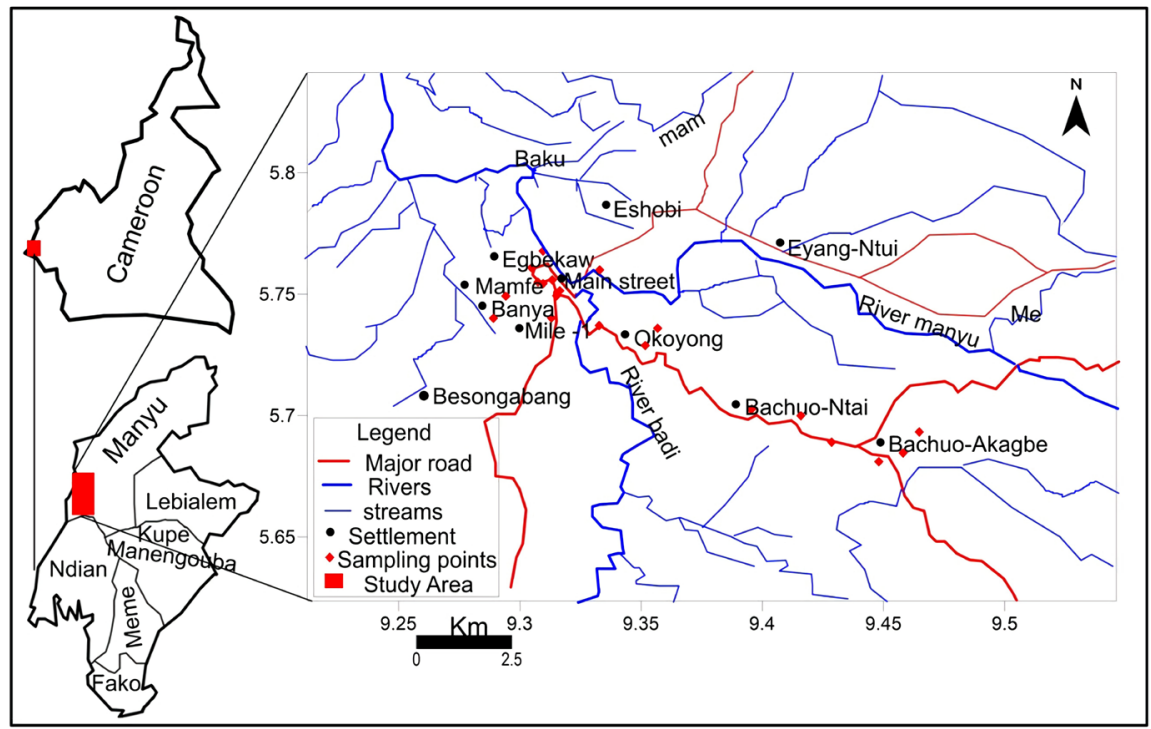

Figure 1. Location map of the study area showing field tested and sampling points. 


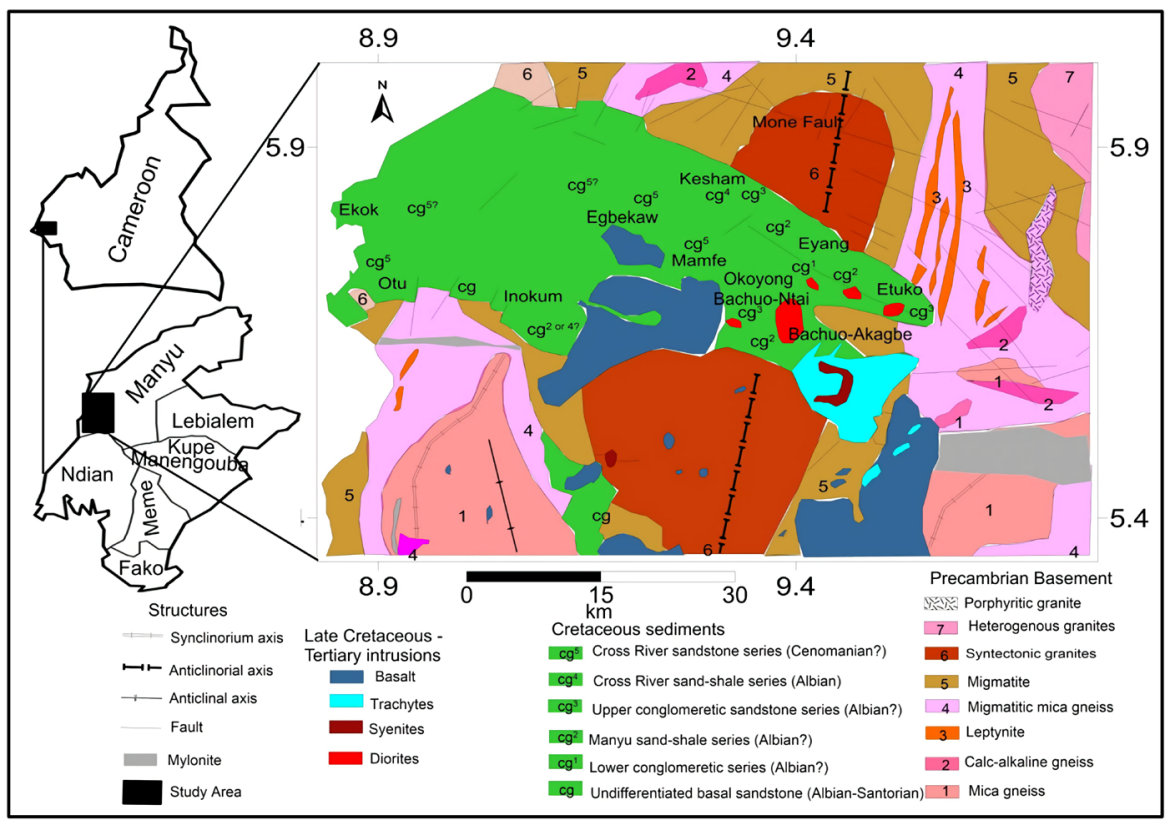

Figure 2. Geologic map of Mamfe (Adapted from Lordon et al., 2017).

the Benue trough and is linked with the West Central African Rift System (CWARS) thought to have formed during the Albian to Cenomanian (Eyong et al., 2013) as a result of basement rifting associated with the reactivation of E-W trending mylonite zones within the Pan-African basement (Dumort, 1968). The basin narrows towards the east and widens towards the west across the Cameroon/Nigeria border into the Benue trough were Albianmarine deposits of Abakaliki Formation outcrops. The basin is fringed by reactivated, fault-bounded granite-gneissic rocks of the Pan-African Mobile Belt (550 $\pm 100 \mathrm{Ma})$ and are both intruded by volcanic rocks (Eyong et al., 2013).

Mamfe Basin lies in a NW-SE trending trough with a length of $130 \mathrm{~km}$ and a width of $60 \mathrm{~km}$ and constitutes a small prolongation of the Benue trough (Nguimbous-Kouoh et al., 2012). Ndougsa-Mbarga et al. (2007) described the Mamfe basin as the smallest of three side rifts associated with the Benue trough of west-central Africa. It extends from the lower Benue trough in Nigeria into Southwestern Cameroon where it narrows and terminates under the Cameroon volcanic line.

The Mamfe basin is bordered to the south by the Oban Massif granito-gneissic Precambrian Basement Complex which separates it from the Rio del Rey Basin and to the North by the Precambrian rocks of the Obudu Massif. To the West the Basin is open and continues as a part of Anambra basin of Nigeria and in the East and Northeast it narrows and terminates under the CVL.

\section{Materials and Methods}

\subsection{Materials}

The field materials and equipment used in the study are listed in Table 1. 
Table 1. Field equipment, specifications and functions.

\begin{tabular}{|c|c|c|}
\hline Equipment/Softwares & Specifications & Functions \\
\hline Bike & Commercial bikes (Bensikin) & To transport fieldworkers to wells \\
\hline GPS & GARMIN GPSMAP 60CSx & To measure longitude, latitude and elevation of wells \\
\hline EC Meter & HANNA HI 98304/HI98303 & To measure Electrical Conductivity of water. \\
\hline $\mathrm{pH}$ Meter & HANNA HI 98127/HI98107 & To measure $\mathrm{pH}$ of water. \\
\hline Measuring Tape & Weighted measuring tape & Measurement of well diameter and depth. \\
\hline Digital Thermometer & Extech $39240\left(-50^{\circ} \mathrm{C}\right.$ to $\left.200^{\circ} \mathrm{C}\right)$ & To measure temperature of water \\
\hline Total Dissolved Solid meter & Hanna HI 96301 with ATC & To measure Total dissolved solids in water \\
\hline Water sampler & Gallenkampf $1000 \mathrm{ml}$ & To collect well water sample from well \\
\hline ArcGIS & Version 10.1 & GIS Drawing sampling/Tests location maps \\
\hline Global Mapper & Version 15 & GIS Geolocation of wells \\
\hline Surfer Golden Software & Version 12 & GIS plotting contours for spatial distribution \\
\hline AqQA/Aquachem & Version 15 & For the analysis/interpretation of water chemistry \\
\hline
\end{tabular}

\subsection{Methods}

A reconnaissance survey was carried out to identify wells, springs and streams in June 2016 as per ISO 5667-1 (2006). Seasonal tests/measurements were carried out in September 2016 wet season and Dry season February 2017 respectively. 53 dug wells, were measured/tested in situ for: coordinates of wells, Surface elevation, Well water level, Dug wells depths well diameter, Electrical conductivity (EC), $\mathrm{pH}$, Total dissolved solids (TDS) and Temperature $\left({ }^{\circ} \mathrm{C}\right)$. Forty (40) groundwater samples 20 in wet and dry seasons were collected in a high density polyethylene (HPDE) $500 \mathrm{ml}$ bottles sealed and sent to the laboratory as per sampling protocols; ISO 5667-3 (2003), ISO 5667-11 (2009) using the standard methods APHA (1995) to analyze for:

1) Major cations in mg/L: $\mathrm{Ca}^{2+}, \mathrm{Mg}^{2+}, \mathrm{Na}^{+}, \mathrm{K}^{+}$and $\mathrm{NH}_{4}^{+}$.

2) Major anions in $\mathrm{mg} / \mathrm{L}: \mathrm{HCO}_{3}^{-}, \mathrm{Cl}^{-}, \mathrm{SO}_{4}^{2-}, \mathrm{HPO}_{4}^{2-}$ and $\mathrm{NO}_{3}^{-}$

Ionic ratio for indicative elements is a useful hydrogeochemical tool to identify source rock of ions and formation contribution to solute hydrogeochemistry Hounslow (1995). These were used in this study.

Gibbs Diagram is a plot of $\mathrm{Na}^{+} /\left(\mathrm{Na}^{+}+\mathrm{HCO}_{3}^{-} \mathrm{Ca}^{2+}\right)$ and $\mathrm{Cl}^{-} /\left(\mathrm{Cl}+\mathrm{HCO}_{3}^{-}\right)$as a function of TDS are widely employed to determine the sources of dissolved geochemical constituents. These plots reveal the relationships between water composition and the three main hydrogeochemical processes involved in ions acquisition; Atmospheric precipitation, rock weathering or evaporation crystallisation.

Pipers Diagram is a graphical representation of the chemistry of water sample on three fields; the cation ternary field with $\mathrm{Ca}, \mathrm{Mg}$ and $\mathrm{Na}+$ Kapices, the anion ternary field with $\mathrm{HCO}_{3}, \mathrm{SO}_{4}$ and $\mathrm{Cl}^{-}$apices. These two fields are projected onto 
a third diamond field. The diamond field is a matrix transformation of the graph of the anions [sulfate + chloride] $/ \Sigma$ anions and cations $[\mathrm{Na}+\mathrm{K}] / \Sigma$ cations. This plot is a useful hydrogeochemical tool to compare water samples, determine water type and hydrogeochemical facies Langguth (1966). This has been used here for these purposes.

Durov diagram is a composite plot consisting of two ternary diagrams where the milliequivalent percentages of cations are plotted perpendicularly against those of anions; the sides of the triangles form a central rectangular binary plot of total cation vs. total anion concentrations. These are divided into nine classes by Lloyd and Heathcoat (1985) which give the hydrogeochemical processes determining the character of the water types in the aquiferous formation Langguth (1966).

WQI was calculated by adopting Weighted Arithmetical Index method considering thirteen water quality parameters $(\mathrm{pH}, \mathrm{EC}, \mathrm{TDS}$, total alkalinity, total hardness, $\mathrm{Ca}^{2+}, \mathrm{Mg}^{2+}, \mathrm{Na}^{+}, \mathrm{K}^{+}, \mathrm{Cl}^{-}, \mathrm{SO}_{4}^{2-}, \mathrm{NO}_{3}^{-}, \mathrm{NH}_{4}^{+}$) in order to assess the degree of groundwater contamination and suitability Table 2 .

For Agro-industrial suitability the following parameters were used; sodium adsorption ratio SAR, permeability index PI, Magnesium adsorption ratio MAR, percent sodium \%Na, Kelly's ratio KR and Residual sodium carbonate RSC and Wilcox diagram Table 2.

The following sofwares; Surfer 12, Global mapper 11 and AqQA 1.5 AGIS 10.3 were used for data presentation, interpretation and analysis.

\section{Results and Interpretation}

\subsection{Physicochemical Parameters}

The physicochemical parameters groundwater in Mamfe: Temperature, $\mathrm{pH}$, EC

Table 2. Indices used in the calculation of water quality and irrigation water quality.

\begin{tabular}{|c|c|c|}
\hline & Formula & Reference \\
\hline Percentage Sodium & $\% \mathrm{Na}=\frac{\mathrm{Na}^{+}+\mathrm{K}^{+}}{\mathrm{Na}^{+}+\mathrm{K}^{+}+\mathrm{Ca}^{2+}+\mathrm{Mg}^{2+}} \times 100$ & Wilcox (1955) \\
\hline Kelly Ratio & $\mathrm{KR}=\frac{\mathrm{Na}^{+}}{\mathrm{Ca}^{2+}+\mathrm{Mg}^{2+}}$ & Kelley (1940) \\
\hline $\begin{array}{c}\text { Magnesium Absorption } \\
\text { Ratio }\end{array}$ & $\operatorname{MAR}=\left(\frac{\mathrm{Mg}^{2+}}{\mathrm{Mg}^{2+}+\mathrm{Ca}^{2+}}\right) \times 100$ & Paliwal (1972) \\
\hline Total Hardness & $\mathrm{TH}\left(\mathrm{CaCO}_{3}\right) \mathrm{mg} / \mathrm{L}=2.5 \mathrm{Ca}^{2+}+4.1 \mathrm{Mg}^{2+}$ & Todd (1980) \\
\hline Residual Sodium Carbonate & $\mathrm{RSC}=\left(\mathrm{CO}_{3}+\mathrm{HCO}_{3}-(\mathrm{Ca}+\mathrm{Mg})\right)$ & Eaton (1950) \\
\hline Sodium Absorption Ratio & $\mathrm{SAR}=\frac{\mathrm{Na}}{\sqrt{\frac{\mathrm{Ca}+\mathrm{Mg}}{2}}}$ & Richards (1954) \\
\hline Permeability Index & $\mathrm{PI}=\frac{\left((\mathrm{Na}+\mathrm{K})+\sqrt{\mathrm{HCO}_{3}}\right) * 100}{\mathrm{Ca}+\mathrm{Mg}+\mathrm{Na}+\mathrm{K}}$ & Doneen (1962) \\
\hline Water Quality Index & $\mathrm{WQI}=\sum_{i=1}^{n} W_{i} q_{i}\left[\sum_{i=1}^{n} W_{i}\right]^{-1}$ & $\begin{array}{l}\text { Sisodia and Moundiotiya } \\
\qquad(2006)\end{array}$ \\
\hline
\end{tabular}


and TDS for 53 wells were evaluated as shown in Table 3. From Table 3 all physicochemical parameters vary with seasons indicating seasonal influence phreatic aquifer.

\section{Water Level Fluctuations}

Depth-to statues water values $(\mathrm{m})$ of groundwater in Mamfe ranged from: 0.5 - 9.5 in the Wet season and 0.5 - 14.5 in the dry season Figure 3.

\section{Groundwater flow direction}

Groundwater flows towards the Northwestern part of the study area during the wet season and dry season but during the dry season some water flows towards Bachuo-Akagbe Figure 4.

\section{Temperature}

Temperature values ${ }^{\circ} \mathrm{C}$ of Mamfe groundwater ranged from: 24.4 - 30.1 wet season $25^{\circ} \mathrm{C}-38.6^{\circ} \mathrm{C}$ Figure 5 .

$\mathrm{pH}$

The $\mathrm{pH}$ value of most of the groundwater samples in the study area ranged from $4.3-8.6$ in the wet season and $5.5-9.3$ in the dry season Figure 6. This

Table 3. Basic Statistics of the physicochemical parameters found in groundwater, min, max, mean and standard deviation of these elements in both the wet season and dry season.

\begin{tabular}{ccccccccc}
\hline Parameters & \multicolumn{9}{c}{ Wet } & \multicolumn{5}{c}{ Dry } \\
\hline & Min & Max & Mean & Std. & Min & Max & Mean & Std. \\
\hline $\mathrm{T}\left({ }^{\circ} \mathrm{C}\right)$ & 24.4 & 30.1 & 27.77 & 0.98 & 25 & 38.6 & 28.04 & 2.46 \\
$\mathrm{PH}$ & 4.3 & 8.6 & 6.11 & 0.87 & 5.5 & 9.3 & 7.53 & 0.75 \\
$\mathrm{EC}(\mu \mathrm{S} / \mathrm{cm})$ & 3 & 1348 & 178.9 & 202.5 & 6.0 & 944 & 174.89 & 162.03 \\
$\mathrm{TDS}(\mathrm{mg} / \mathrm{L})$ & 2 & 903.16 & 119.89 & 135.65 & 4.02 & 632.48 & 100.13 & 108.52 \\
\hline
\end{tabular}

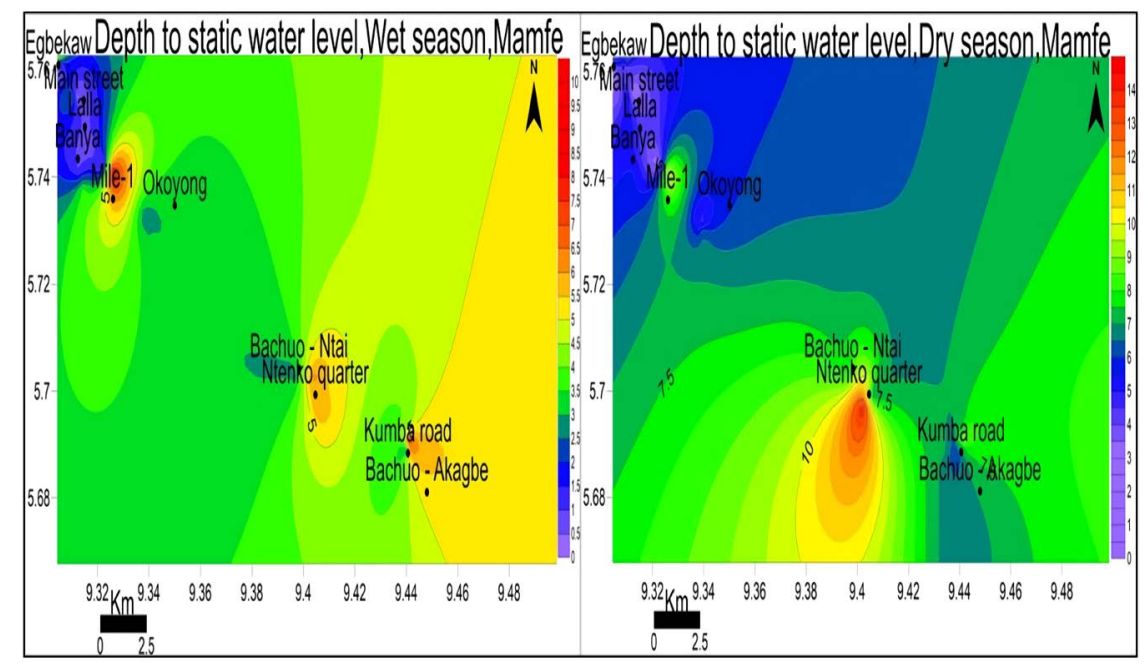

Figure 3. Depth to static water level in Mamfe (a) wet season (b) dry season. Note high water level is recorded during the dry season than in the wet season. High values are atBachuo-Ntai, Mile-1 and Bachuo-Akagbe in the wet season with high values in Mile-1 during the dry season. 


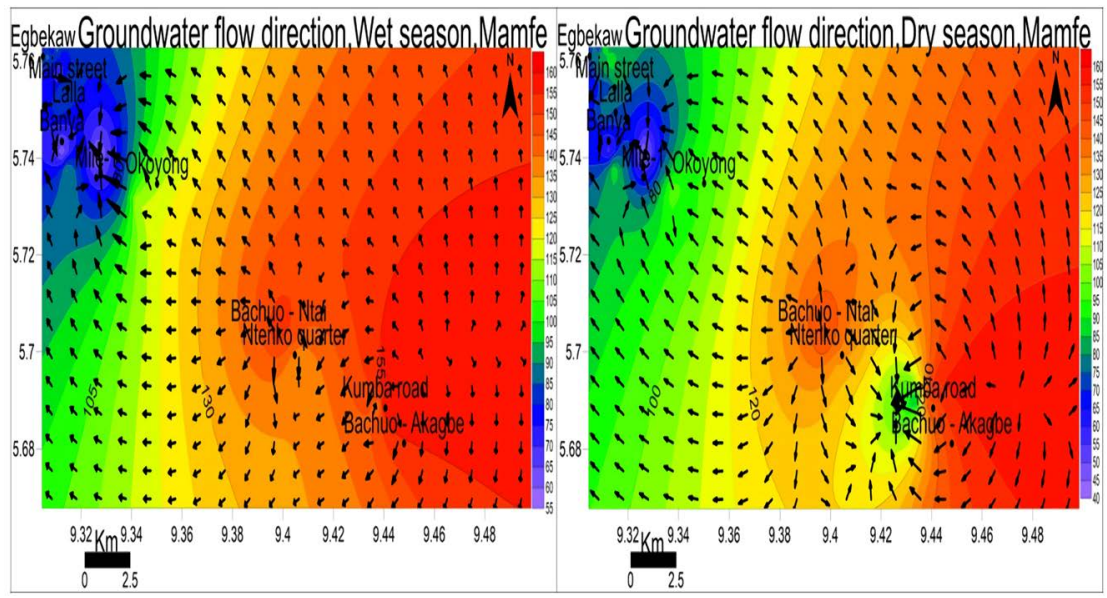

Figure 4. Groundwater flow direction in Mamfe indicating that water flows towards the Northwestern part of the study area that is towards mile-1but during the dry season some water flows towards Bachuo-Akagbe indicating that it is a recharge zone.

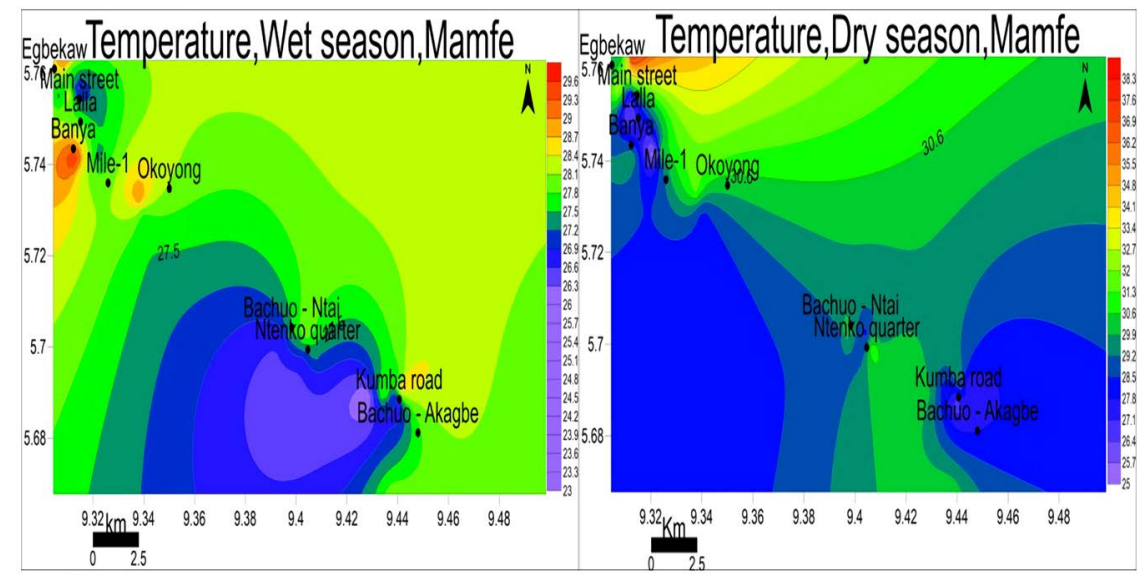

Figure 5. Variation of Mamfe groundwater temperatures. Temperatures are generally higher in the dry season and lower in the wet season. High temperatures are in Banya, Egbekaw and Okoyong while low value are in Bachuo-Akagbe and Bachuo-Ntaiin the wet season and during the dry season the highest values are found Egbekaw and Main Street.

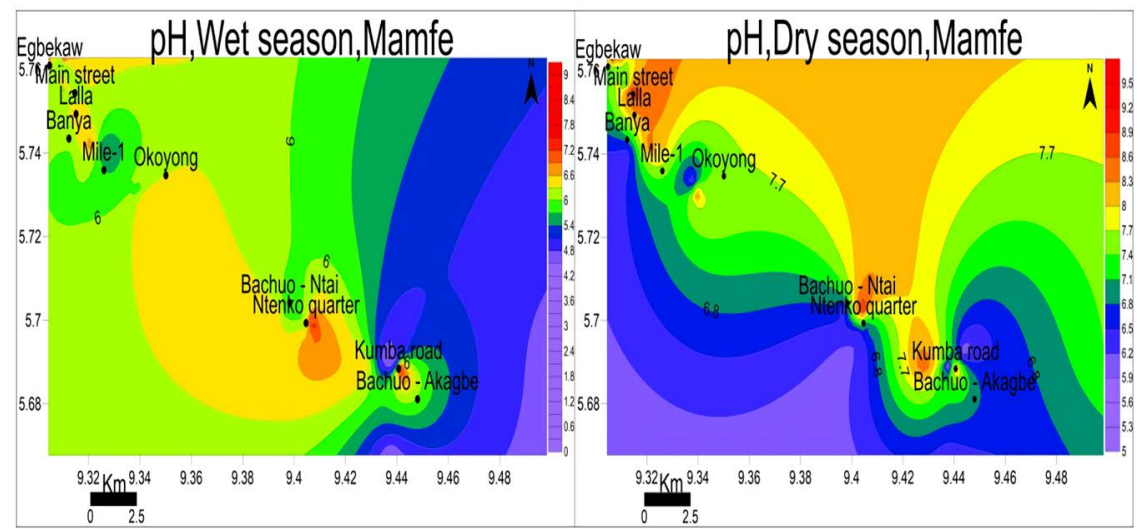

Figure 6. Spatial variation of dug well water $\mathrm{pH}$ in Mamfe during (a) wet season (b) dry season; Note decrease in $\mathrm{pH}$ values wet season around Bachuo-Akagbe while in the dry seasons the $\mathrm{pH}$ values increase around Main Street, Lalla and Bachuo-Ntai. 
clearly shows that the groundwater in the study area is slightly acidic to alkaline in both seasons.

\section{Electrical conductivity (EC)}

The EC ranges from $3-1348 \mu \mathrm{S} / \mathrm{cm}$ during the wet season and $6-944 \mu \mathrm{S} / \mathrm{cm}$ during the dry season Figure 7.

The high electrical conductivity is due to high solute concentration in water.

\section{Total dissolved solids (TDS)}

The total dissolved solids range from $2.0-903.16 \mathrm{mg} / \mathrm{L}$ in the wet season and $4.02-632.48 \mathrm{mg} / \mathrm{L}$ in the dry season Figure 8.

\subsection{Chemical Parameters of Groundwater}

The results of the chemical analysis varied in both seasons Table 4(a) and Table 4(b) (Figure 9 \& Figure 10).

\section{Mechanism controlling water chemistry}

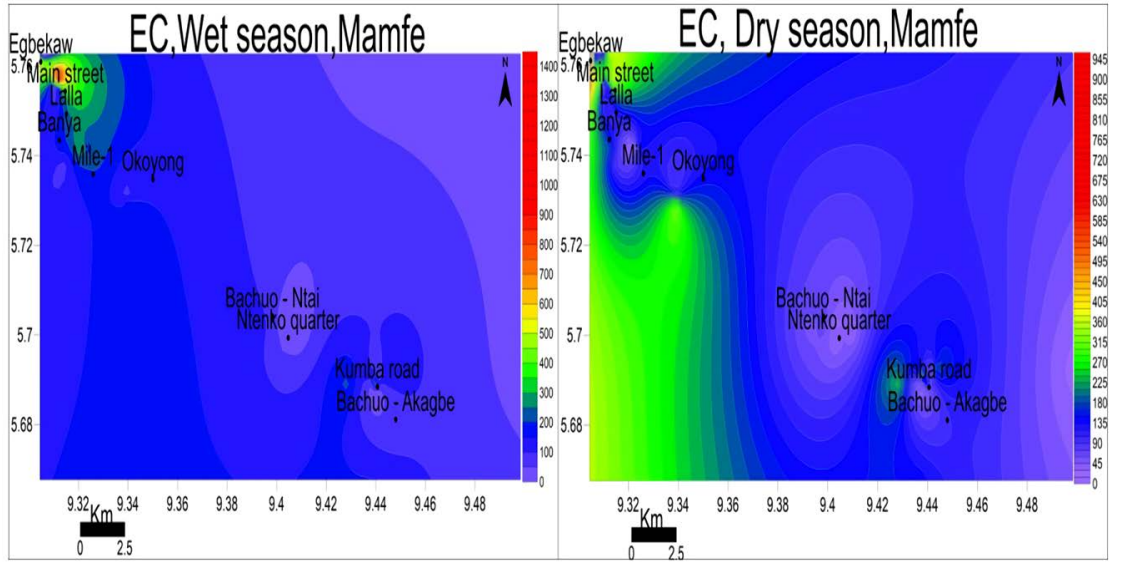

Figure 7. Spatial variation of dug well water Electrical Conductivities $(\mu S)$ in Mamfe during (a) wet season (b) dry season; EC is at maximum in the wet season and minimum in the dry season.

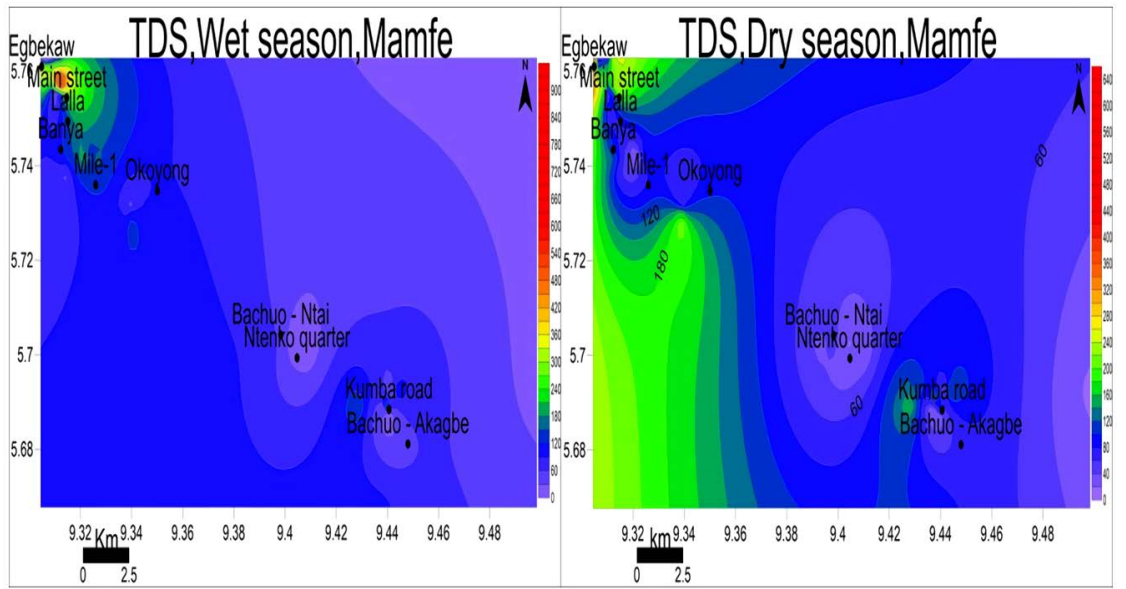

Figure 8. Spatial variation of dug well water total dissolved solids mg/L in Mamfe during wet and dry season. TDS is highest in the wet season and lowest in the dry season. In the wet season the highest value is at Main Street. 

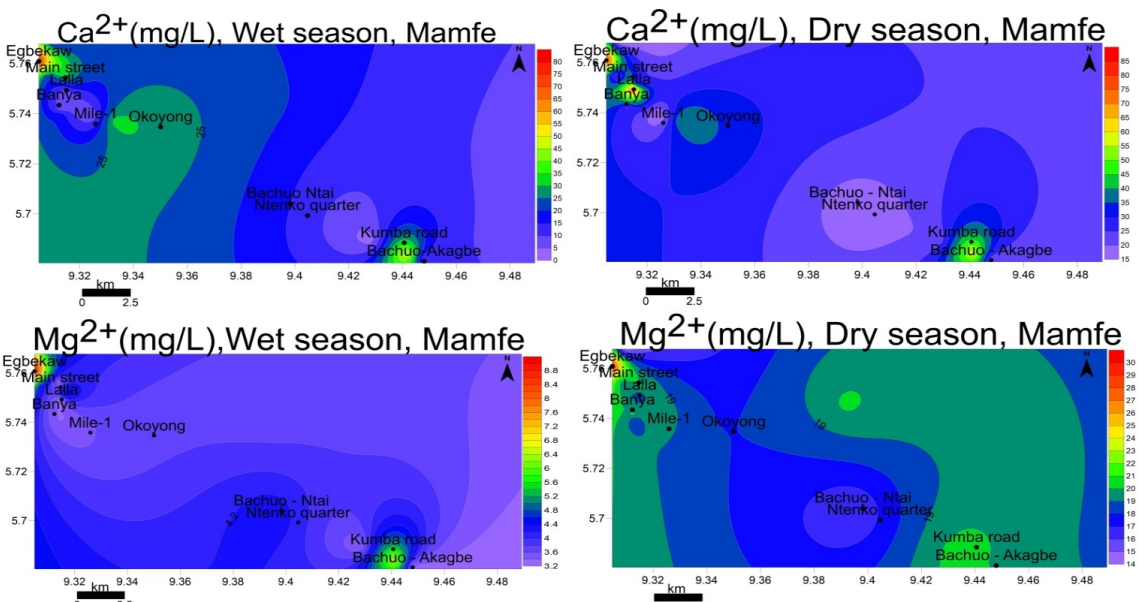

$\mathrm{Na}^{+}(\mathrm{mg} / \mathrm{L})$, Wet season, Mamfe

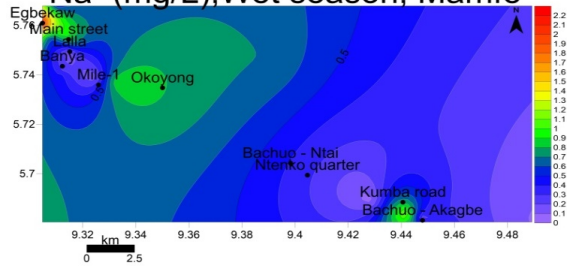

$\mathrm{Na}^{+}(\mathrm{mg} / \mathrm{L})$. Dry season. Mamfe

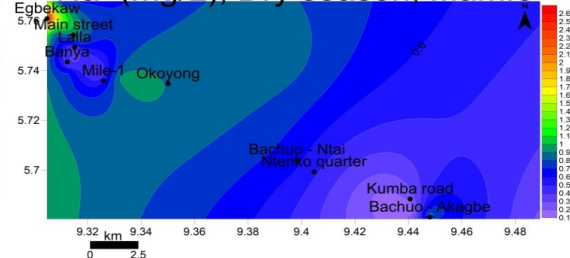

$\mathrm{K}^{+}(\mathrm{mg} / \mathrm{L})$, Wet season, Mamfe

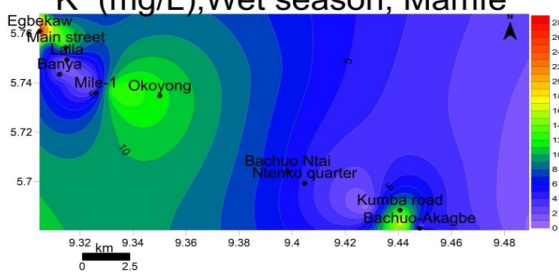

$\mathrm{K}^{+}(\mathrm{mg} / \mathrm{L})$,Dry season, Mamfe

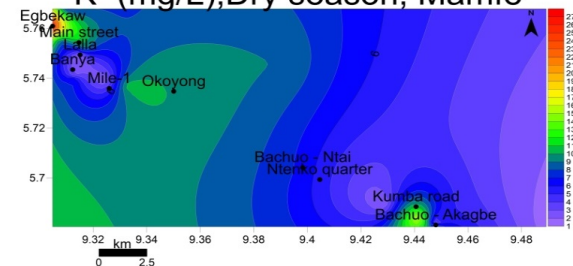

$\mathrm{NH}_{4}^{+}(\mathrm{mg} / \mathrm{L})$, Wet season, Mamfe

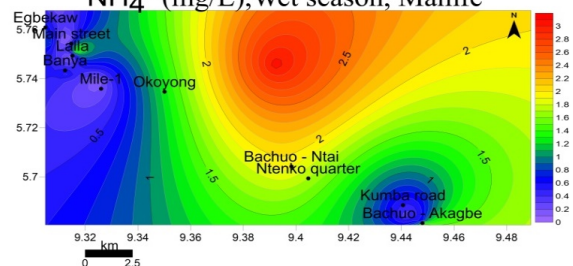

Figure 9. Spatial distribution of Cations; $\mathrm{Ca}^{2+}, \mathrm{Mg}^{2+}, \mathrm{Na}, \mathrm{K}^{+}, \mathrm{NH}_{4}^{+}$in Mamfe Groundwater showing variations for both wet and dry seasons.

Table 4. (a) Results of chemical analysis during wet season: The values of rainwater, springs, streams, Rivers and groundwater are similar indicating connectivity typical of recharge zones in phreatic aquifers; (b) Results of chemical analysis during dry season: The values of rainwater, springs, streams, Rivers and groundwater are similar indicating connectivity typical of recharge zones in phreatic aquifers.

(a)

\begin{tabular}{cccccccccccc}
\hline \multicolumn{10}{c}{ Wet Season $(\mathrm{mg} / \mathrm{L})$} \\
\hline $\mathrm{SN}$ & Names & $\mathrm{Na}^{+}$ & $\mathrm{K}^{+}$ & $\mathrm{Ca}^{2+}$ & $\mathrm{Mg}^{2+}$ & $\mathrm{NH}_{4}^{+}$ & $\mathrm{HPO}_{4}^{2-}$ & $\mathrm{NO}_{3}^{-}$ & $\mathrm{SO}_{4}^{2-}$ & $\mathrm{CL}^{-}$ & $\mathrm{HPO}_{4}^{2-}$ \\
\hline 1 & Bachuo-Akagbe 1 & 0.51 & 7.45 & 18.6 & 3.41 & 1.76 & 34.16 & 0.01 & 1.55 & 16.00 & 0 \\
2 & Bachuo-Akagbe 2 & 0.09 & 0.86 & 5.40 & 3.22 & 1.71 & 3.66 & 0.00 & 1.46 & 36.00 & 0.06 \\
3 & Bachuo-Akagbe 3 & 0.15 & 1.64 & 5.40 & 3.22 & 0.65 & 6.10 & 0.00 & 1.50 & 10.00 & 0.05 \\
\hline
\end{tabular}




\section{Continued}

\begin{tabular}{|c|c|c|c|c|c|c|c|c|c|c|c|}
\hline 4 & Bachuo-Ntai 1 & 1.17 & 18.17 & 50.6 & 6.64 & 0.35 & 15.86 & 0.01 & 4.09 & 28.00 & 0.00 \\
\hline 5 & Bachuo-Ntai 2 & 0.06 & 0.51 & 2.66 & 3.41 & 0.94 & 4.88 & 0.00 & 1.74 & 0.00 & 0.00 \\
\hline 6 & Bachuo-Ntai 3 & 0.41 & 6.2 & 15.98 & 4.39 & 1.91 & 0.00 & 0.00 & 1.97 & 8.00 & 0.36 \\
\hline 7 & Okoyong 1 & 0.90 & 13.26 & 32 & 3.71 & 0.53 & 3.66 & 0.00 & 2.72 & 14.00 & 0.00 \\
\hline 8 & Mile-1 & 0.69 & 7.41 & 21.4 & 3.61 & 3.06 & 0.00 & 0.00 & 3.38 & 26.00 & 0.00 \\
\hline 9 & Garri quarter & 0.62 & 6.83 & 21.4 & 4.97 & 1.59 & 28.06 & 0.02 & 5.64 & 20.00 & 0.07 \\
\hline 10 & Banya & 0.41 & 4.76 & 18.6 & 3.41 & 0.41 & 28.06 & 0.01 & 5.73 & 10.00 & 0.00 \\
\hline 11 & New layout 1 & 0.62 & 6.83 & 21.4 & 4.19 & 0.82 & 10.98 & 0.01 & 2.44 & 36.00 & 0.02 \\
\hline 12 & New layout 2 & 0.62 & 6.83 & 21.4 & 4.28 & 0.24 & 14.64 & 0.01 & 3.71 & 32.00 & 0.00 \\
\hline 13 & Small Mamfe & 0.3 & 3.32 & 8.00 & 3.41 & 0.71 & 4.88 & 0.00 & 2.54 & 6.00 & 0.04 \\
\hline 14 & Tanjong Street & 0.97 & 13.49 & 34.6 & 3.22 & 0.00 & 7.32 & 0.00 & 6.72 & 18.00 & 0.00 \\
\hline 15 & Lalla & 0.14 & 1.01 & 8.00 & 3.31 & 0.12 & 0.00 & 0.00 & 1.93 & 10.00 & 0.06 \\
\hline 16 & Egbekaw 1 & 0.51 & 5.69 & 18.6 & 5.08 & 0.24 & 37.82 & 0.01 & 7.99 & 14.00 & 0.00 \\
\hline 17 & Egbekaw 2 & 2.23 & 29.52 & 82.6 & 9.36 & 0.71 & 18.3 & 0.01 & 3.76 & 96.00 & 0.00 \\
\hline 18 & Spring & 0.18 & 1.13 & 8.00 & 3.31 & 0.35 & 10.98 & 0.01 & 2.44 & 0.00 & 0.00 \\
\hline 19 & River Badi & 0.14 & 1.13 & 5.32 & 3.52 & 0.11 & 8.54 & 0.00 & 2.82 & 0.00 & 0.00 \\
\hline \multirow[t]{5}{*}{20} & Rain water & 0.28 & 2.61 & 10.66 & 2.93 & 0.00 & 2.44 & 0.00 & 1.60 & 4.00 & 0.00 \\
\hline & Min & 0.06 & 0.51 & 2.66 & 2.93 & 0.00 & 0.00 & 0.00 & 1.46 & 0.00 & 0.00 \\
\hline & $\operatorname{Max}$ & 2.23 & 29.52 & 82.60 & 9.36 & 3.06 & 37.82 & 0.02 & 7.99 & 96.00 & 0.36 \\
\hline & Mean & 0.55 & 6.93 & 20.53 & 4.13 & 0.81 & 12.02 & 0.01 & 3.29 & 19.20 & 0.03 \\
\hline & Std. & 0.50 & 7.13 & 18.78 & 1.51 & 0.80 & 11.63 & 0.01 & 1.88 & 21.41 & 0.08 \\
\hline
\end{tabular}

(b)

\begin{tabular}{|c|c|c|c|c|c|c|c|c|c|c|c|}
\hline \multicolumn{12}{|c|}{ Dry Season $(\mathrm{mg} / \mathrm{L})$} \\
\hline SN & Names & $\mathrm{Na}^{+}$ & $\mathrm{K}^{+}$ & $\mathrm{Ca}^{2+}$ & $\mathrm{Mg}^{2+}$ & $\mathrm{NH}_{4}^{+}$ & $\mathrm{HPO}_{4}^{2-}$ & $\mathrm{NO}_{3}^{-}$ & $\mathrm{SO}_{4}^{2-}$ & $\mathrm{CL}^{-}$ & $\mathrm{HPO}_{4}^{2-}$ \\
\hline 1 & Bachuo-Akagbe 1 & 0.64 & 9.34 & 21.45 & 20.14 & 0.00 & 45.24 & 0.00 & 0.15 & 12.00 & 0.00 \\
\hline 2 & Bachuo-Akagbe 2 & 0.28 & 1.45 & 20.2 & 18.75 & 0.00 & 91.18 & 0.00 & 0.18 & 17.00 & 0.04 \\
\hline 3 & Bachuo-Akagbe 3 & 0.94 & 2.38 & 20.15 & 18.94 & 0.00 & 90.25 & 0.00 & 0.14 & 10.00 & 0.02 \\
\hline 4 & Bachuo-Ntai 1 & 0.13 & 16.23 & 60.14 & 21.1 & 0.00 & 121.05 & 0.00 & 1.42 & 15.00 & 0.00 \\
\hline 5 & Bachuo-Ntai 2 & 0.18 & 1.88 & 21.25 & 20.01 & 0.00 & 51.25 & 0.00 & 0.14 & 0.00 & 0.00 \\
\hline 6 & Bachuo-Ntai 3 & 0.64 & 6.95 & 16.75 & 15.95 & 0.00 & 150.00 & 0.00 & 0.11 & 0.00 & 0.25 \\
\hline 7 & Okoyong 1 & 0.95 & 10.45 & 41.34 & 18.25 & 0.00 & 48.00 & 0.00 & 0.18 & 10.00 & 0.00 \\
\hline 8 & Mile-1 & 0.88 & 8.95 & 28.05 & 20.25 & 0.00 & 120.00 & 0.00 & 1.12 & 16.00 & 0.00 \\
\hline 9 & Garri quarter & 0.85 & 8.81 & 27.45 & 20.34 & 0.00 & 45.00 & 0.01 & 2.24 & 10.00 & 0.04 \\
\hline 10 & Banya & 0.71 & 6.84 & 28.11 & 18.25 & 0.00 & 46.00 & 0.01 & 2.16 & 18.00 & 0.00 \\
\hline 11 & New layout 1 & 0.84 & 8.74 & 25.00 & 19.74 & 0.00 & 37.00 & 0.01 & 0.19 & 0.00 & 0.01 \\
\hline 12 & New layout 2 & 0.83 & 8.46 & 25.00 & 19.63 & 0.00 & 46.00 & 0.01 & 0.21 & 12.00 & 0.00 \\
\hline 13 & Small Mamfe & 0.45 & 2.94 & 17.00 & 20.41 & 0.00 & 23.95 & 0.00 & 1.18 & 10.00 & 0.01 \\
\hline
\end{tabular}


R. A. Il Akoachere et al.

\begin{tabular}{rccccccccccc}
\multicolumn{1}{l}{ Continued } \\
\hline 14 & Tanjong Street & 0.99 & 11.88 & 37.45 & 20.22 & 0.00 & 24.00 & 0.00 & 3.05 & 12.00 & 0.00 \\
15 & Lalla & 0.35 & 2.05 & 80.21 & 18.18 & 0.00 & 91.00 & 0.00 & 0.13 & 94.00 & 0.04 \\
16 & Egbekaw 1 & 0.63 & 7.15 & 16.00 & 14.25 & 0.00 & 101.33 & 0.00 & 4.17 & 0.00 & 0.00 \\
17 & Egbekaw 2 & 2.64 & 28.11 & 85.45 & 30.45 & 0.00 & 100.00 & 0.00 & 1.22 & 0.00 & 0.00 \\
18 & Spring & 0.49 & 2.03 & 16.49 & 22.00 & 0.00 & 132.00 & 0.00 & 0.19 & 0.00 & 0.00 \\
19 & River Badi & 0.37 & 2.05 & 12.94 & 20.00 & 0.00 & 130.00 & 0.00 & 0.18 & 0.00 & 0.00 \\
20 & Rain water & 0.57 & 4.90 & 8.00 & 16.26 & 0.00 & 19.79 & 0.02 & 0.16 & 0.00 & 0.02 \\
& Min & 0.13 & 1.45 & 8.00 & 14.25 & 0.00 & 19.79 & 0.00 & 0.11 & 0.00 & 0.00 \\
& Max & 2.64 & 28.11 & 85.45 & 30.45 & 0.00 & 150.00 & 0.02 & 4.17 & 94.00 & 0.25 \\
& Mean & 0.72 & 7.58 & 30.42 & 19.66 & 0.00 & 75.65 & 0.00 & 0.93 & 11.80 & 0.02 \\
& Std. & 0.52 & 6.29 & 21.26 & 3.15 & 0.00 & 41.40 & 0.01 & 1.16 & 20.49 & 0.06 \\
\hline
\end{tabular}

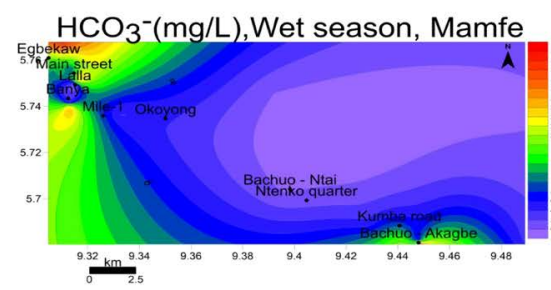

$\mathrm{HCO}_{3}{ }^{-}(\mathrm{mg} / \mathrm{L})$, Dry season, Mamfe

$\mathrm{Cl}^{-}(\mathrm{mg} / \mathrm{L})$, Wet season, Mamfe
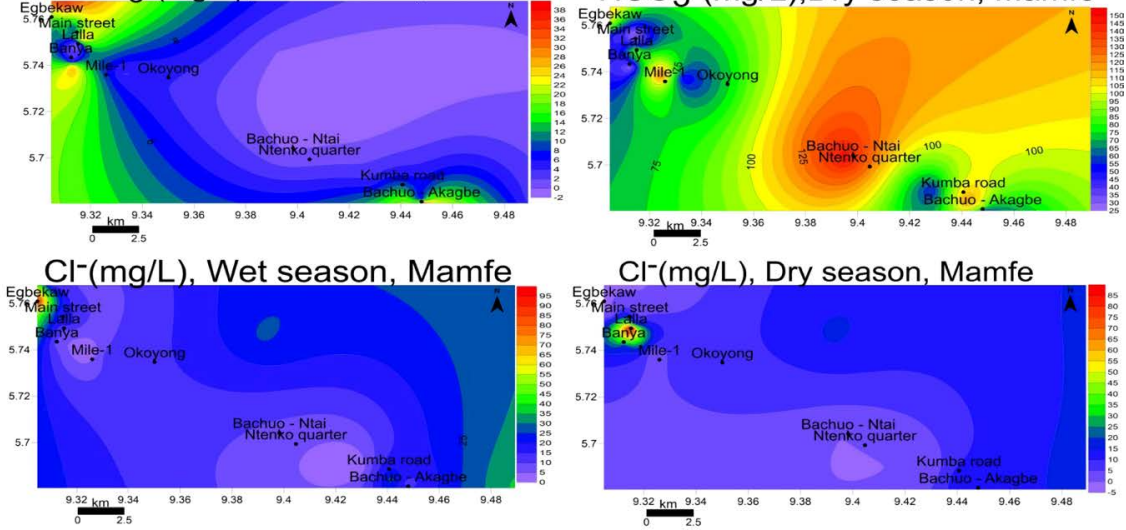

$\mathrm{Cl}^{-}(\mathrm{mg} / \mathrm{L})$, Dry season, Mamfe

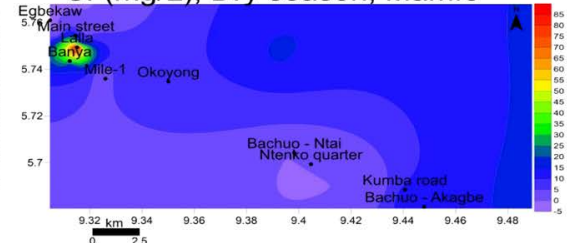

$\mathrm{SO}_{4}{ }^{2-}(\mathrm{mg} / \mathrm{L})$,Wet season, Mamfe

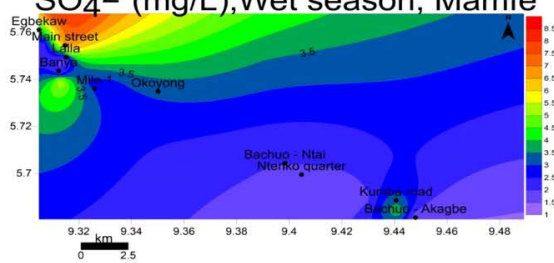

$\mathrm{SO}_{4}{ }^{2-}(\mathrm{mg} / \mathrm{L})$, Dry season, Mamfe

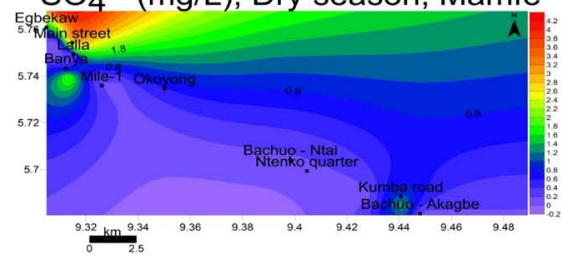

$\mathrm{NO}_{3}{ }^{-}(\mathrm{mg} / \mathrm{L})$, Wet season, Mamfe

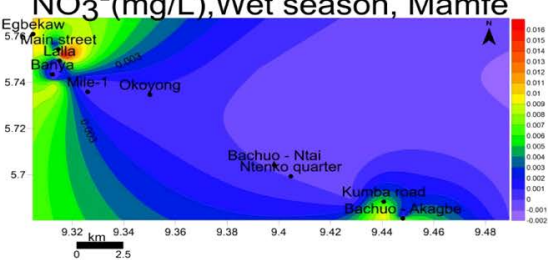

$\mathrm{NO}_{3}{ }^{-}(\mathrm{mg} / \mathrm{L})$, Dry season, Mamfe

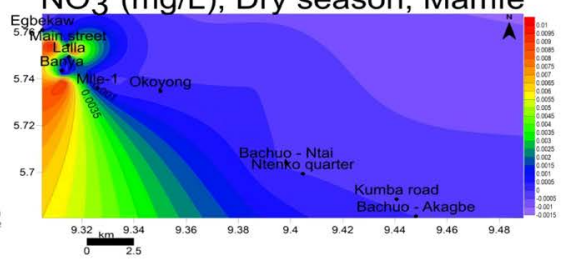

$\mathrm{HPO}_{4}{ }^{2-}(\mathrm{mg} / \mathrm{L})$, Wet season, Mamfe

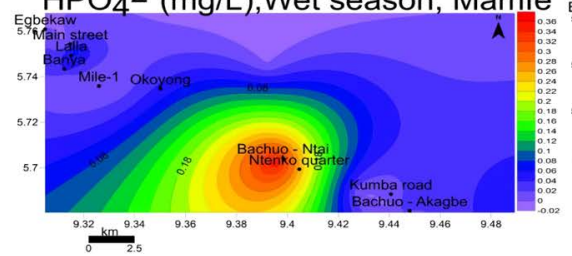

$\mathrm{HPO}_{4}{ }^{2-}(\mathrm{mg} / \mathrm{L})$, Dry season, Mamfe

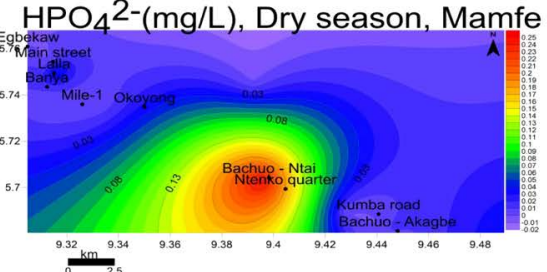

Figure 10. Spatial distribution of Anions; $\mathrm{HCO}_{3}^{-}, \mathrm{CL}^{-}, \mathrm{SO}_{4}^{2-}, \mathrm{HPO}_{4}^{2-}, \mathrm{NO}_{3}^{-}$in Mamfe Groundwater showing variations for both wet and dry seasons. 
Ionic ratios of groundwater in Mamfe

18 ionic ratios in groundwater were used to deduce formation inputs in parts of the Mamfe basin, as follows Tables 5(a)-(c).

Table 5. (a) Ionic ratios groundwater in Mamfe; (b) Ionic ratios groundwater in Mamfe; (c) Ionic ratios for wet and dry seasons with determined formation input.

(a)

\begin{tabular}{|c|c|c|c|c|c|c|c|c|c|c|c|c|c|c|c|c|c|c|}
\hline SN & $\begin{array}{c}\mathrm{SO}_{4} \\
/ \mathrm{Cl}\end{array}$ & $\begin{array}{l}\mathrm{Na} \\
/ \mathrm{Cl}\end{array}$ & $\begin{array}{l}\mathrm{Mg} \\
/ \mathrm{Cl}\end{array}$ & $\begin{array}{c}\mathrm{Na} \\
/ \mathrm{HCO}_{3}\end{array}$ & $\begin{array}{c}\mathrm{Ca} \\
/ \mathrm{HCO}_{3}\end{array}$ & $\begin{array}{c}\mathrm{Ca} \\
/ \mathrm{SO}_{4}\end{array}$ & $\begin{array}{c}\mathrm{Ca} \\
/ \mathrm{Mg}\end{array}$ & $\begin{array}{c}\mathrm{Ca}+ \\
\mathrm{Mg} / \\
\mathrm{Na}+\mathrm{K}\end{array}$ & $\begin{array}{c}\mathrm{HCO}_{3} / \\
\sum \mathrm{An}\end{array}$ & $\begin{array}{l}\mathrm{NO}_{3} / \\
\sum \mathrm{An}\end{array}$ & $\begin{array}{l}\mathrm{SO}_{4} / \\
\sum \mathrm{An}\end{array}$ & $\begin{array}{l}\mathrm{Mg} \\
/ \mathrm{Ca}\end{array}$ & $\begin{array}{c}\mathrm{Na} \\
/ \mathrm{Na}+ \\
\mathrm{Cl}\end{array}$ & $\begin{array}{c}\mathrm{Mg} / \\
\mathrm{Ca}+ \\
\mathrm{Mg}\end{array}$ & $\begin{array}{c}\mathrm{Ca} \\
/ \mathrm{Ca}+ \\
\left.\mathrm{SO}_{4}\right) \\
\end{array}$ & $\begin{array}{l}\mathrm{Ca}+ \\
\mathrm{Mg} \\
\mathrm{SO}_{4} \\
\end{array}$ & $\begin{array}{c}\mathrm{Cl} \\
/ \Sigma \mathrm{An}\end{array}$ & $\begin{array}{c}\mathrm{Na}+\mathrm{K}-\mathrm{Cl} \\
/ \mathrm{Na}+\mathrm{K}+ \\
\mathrm{Cl}-\mathrm{Ca} \\
\end{array}$ \\
\hline 1 & 0.10 & 0.03 & 0.21 & 0.01 & 0.54 & 12.00 & 0.00 & 0.00 & 0.05 & 0.00 & 0.00 & 0.18 & 0.03 & 0.15 & 0.92 & 14.20 & 0.02 & 0.30 \\
\hline 2 & 0.04 & 0.00 & 0.09 & 0.02 & 1.48 & 3.70 & 1.68 & 9.07 & 0.01 & 0.00 & 0.00 & 0.60 & 0.00 & 0.37 & 0.79 & 5.90 & 0.05 & 0.87 \\
\hline 3 & 0.15 & 0.02 & 0.32 & 0.02 & 0.89 & 3.60 & 1.68 & 4.82 & 0.01 & 0.00 & 0.00 & 0.60 & 0.01 & 0.37 & 0.78 & 5.75 & 0.01 & 0.60 \\
\hline 4 & 0.15 & 0.04 & 0.24 & 0.07 & 3.19 & 12.37 & 7.62 & 2.96 & 0.02 & 0.00 & 0.01 & 0.13 & 0.04 & 0.12 & 0.93 & 14.00 & 0.04 & 0.15 \\
\hline 5 & 0.00 & 0.00 & 0.00 & 0.01 & 0.55 & 1.53 & 0.78 & 10.65 & 0.01 & 0.00 & 0.00 & 1.28 & 1.00 & 0.56 & 0.60 & 3.49 & 0.00 & -0.27 \\
\hline 6 & 0.25 & 0.05 & 0.55 & 0.00 & 0.00 & 0.00 & 0.00 & 0.00 & 0.00 & 0.00 & 0.00 & 0.27 & 0.05 & 0.22 & 0.89 & 10.34 & 0.01 & 0.08 \\
\hline 7 & 0.19 & 0.06 & 0.27 & 0.25 & 8.74 & 0.00 & 8.63 & 2.52 & 0.01 & 0.00 & 0.00 & 0.12 & 0.06 & 0.10 & 0.92 & 13.13 & 0.02 & -0.01 \\
\hline 8 & 0.13 & 0.03 & 0.14 & 0.00 & 0.00 & 6.33 & 0.00 & 3.09 & 0.00 & 0.00 & 0.00 & 0.17 & 0.03 & 0.14 & 0.86 & 7.40 & 0.04 & 0.46 \\
\hline 9 & 0.28 & 0.03 & 0.25 & 0.02 & 0.76 & 3.79 & 4.31 & 3.54 & 0.04 & 0.00 & 0.01 & 0.23 & 0.03 & 0.19 & 0.79 & 4.68 & 0.03 & 0.37 \\
\hline 10 & 0.57 & 0.04 & 0.34 & 0.01 & 0.66 & 3.25 & 0.00 & 0.00 & 0.04 & 0.00 & 0.01 & 0.18 & 0.04 & 0.15 & 0.76 & 3.84 & 0.01 & 0.21 \\
\hline 11 & 0.07 & 0.02 & 0.12 & 0.06 & 1.95 & 0.00 & 5.11 & 3.43 & 0.02 & 0.00 & 0.00 & 0.20 & 0.02 & 0.16 & 0.90 & 10.49 & 0.05 & 0.57 \\
\hline 12 & 0.12 & 0.02 & 0.13 & 0.04 & 1.46 & 0.00 & 5.00 & 3.45 & 0.02 & 0.00 & 0.01 & 0.20 & 0.02 & 0.17 & 0.85 & 6.92 & 0.05 & 0.53 \\
\hline 13 & 0.42 & 0.05 & 0.57 & 0.06 & 1.64 & 3.15 & 2.35 & 0.00 & 0.01 & 0.00 & 0.00 & 0.43 & 0.05 & 0.30 & 0.76 & 4.49 & 0.01 & 0.23 \\
\hline 14 & 0.37 & 0.05 & 0.18 & 0.13 & 4.73 & 0.00 & 10.75 & 0.00 & 0.01 & 0.00 & 0.01 & 0.09 & 0.05 & 0.09 & 0.84 & 5.63 & 0.03 & 0.09 \\
\hline 15 & 0.19 & 0.01 & 0.33 & 0.00 & 0.00 & 4.15 & 2.42 & 9.83 & 0.00 & 0.00 & 0.00 & 0.41 & 0.01 & 0.29 & 0.81 & 5.86 & 0.01 & 0.53 \\
\hline 16 & 0.57 & 0.04 & 0.36 & 0.01 & 0.49 & 2.33 & 3.66 & 3.82 & 0.05 & 0.00 & 0.01 & 0.27 & 0.04 & 0.21 & 0.70 & 2.96 & 0.02 & 0.30 \\
\hline 17 & 0.04 & 0.02 & 0.10 & 0.00 & 0.00 & 0.00 & 8.82 & 0.00 & 0.03 & 0.00 & 0.01 & 0.11 & 0.02 & 0.10 & 0.96 & 24.46 & 0.14 & 0.44 \\
\hline 18 & 0.00 & 0.00 & 0.00 & 0.02 & 0.73 & 3.28 & 2.42 & 8.63 & 0.02 & 0.00 & 0.00 & 0.41 & 1.00 & 0.29 & 0.77 & 4.64 & 0.00 & -0.20 \\
\hline 19 & 0.00 & 0.00 & 0.00 & 0.02 & 0.62 & 1.89 & 1.51 & 6.96 & 0.01 & 0.00 & 0.00 & 0.66 & 1.00 & 0.40 & 0.65 & 3.13 & 0.00 & -0.31 \\
\hline 20 & 0.40 & 0.07 & 0.73 & 0.11 & 4.37 & 6.66 & 3.64 & 4.70 & 0.00 & 0.00 & 0.00 & 0.27 & 0.07 & 0.22 & 0.87 & 8.49 & 0.01 & 0.09 \\
\hline Min & 0.00 & 0.00 & 0.00 & 0.00 & 0.00 & 0.00 & 0.00 & 0.00 & 0.00 & 0.00 & 0.00 & 0.09 & 0.00 & 0.09 & 0.60 & 2.96 & 0.00 & -0.31 \\
\hline $\operatorname{Max}$ & 0.57 & 0.07 & 0.73 & 0.25 & 8.74 & 12.37 & 10.75 & 10.65 & 0.05 & 0.00 & 0.01 & 1.28 & 1.00 & 0.56 & 0.96 & 24.46 & 0.14 & 0.87 \\
\hline Mean & 0.20 & 0.03 & 0.25 & 0.04 & 1.64 & 3.40 & 3.52 & 3.87 & 0.02 & 0.00 & 0.00 & 0.34 & 0.18 & 0.23 & 0.82 & 7.99 & 0.03 & 0.25 \\
\hline$S t d$. & 0.18 & 0.02 & 0.20 & 0.06 & 2.16 & 3.63 & 3.25 & 3.52 & 0.02 & 0.00 & 0.00 & 0.28 & 0.35 & 0.12 & 0.09 & 5.26 & 0.03 & 0.31 \\
\hline
\end{tabular}

(b)

\begin{tabular}{|c|c|c|c|c|c|c|c|c|c|c|c|c|c|c|c|c|c|c|}
\hline $\mathrm{SN}$ & $\begin{array}{c}\mathrm{SO}_{4} \\
/ \mathrm{Cl}\end{array}$ & $\begin{array}{l}\mathrm{Na} \\
/ \mathrm{Cl}\end{array}$ & $\begin{array}{l}\mathrm{Mg} \\
/ \mathrm{Cl}\end{array}$ & $\begin{array}{c}\mathrm{Na} \\
/ \mathrm{HCO}_{3}\end{array}$ & $\begin{array}{c}\mathrm{Ca} \\
/ \mathrm{HCO}_{3}\end{array}$ & $\begin{array}{c}\mathrm{Ca} \\
/ \mathrm{SO}_{4}\end{array}$ & $\begin{array}{c}\mathrm{Ca} \\
/ \mathrm{Mg}\end{array}$ & $\begin{array}{c}\mathrm{Ca}+ \\
\mathrm{Mg} / \\
\mathrm{Na}+\mathrm{K}\end{array}$ & $\begin{array}{c}\mathrm{HCO}_{3} / \\
\sum \mathrm{An}\end{array}$ & $\begin{array}{l}\mathrm{NO}_{3} / \\
\sum \mathrm{An}\end{array}$ & $\begin{array}{l}\mathrm{SO}_{4} / \\
\sum \mathrm{An}\end{array}$ & $\begin{array}{l}\mathrm{Mg} \\
/ \mathrm{Ca}\end{array}$ & $\begin{array}{c}\mathrm{Na} \\
/ \mathrm{Na}+ \\
\mathrm{Cl}\end{array}$ & $\begin{array}{c}\mathrm{Mg} \\
/ \mathrm{Ca}+ \\
\mathrm{Mg}\end{array}$ & $\begin{array}{c}\mathrm{Ca} \\
/ \mathrm{Ca}+ \\
\mathrm{SO}_{4} \\
\end{array}$ & $\begin{array}{c}\mathrm{Ca}+ \\
\mathrm{Mg} \\
/ \mathrm{SO}_{4}\end{array}$ & $\begin{array}{c}\mathrm{Cl} \\
/ \Sigma \mathrm{An}\end{array}$ & $\begin{array}{c}\mathrm{Na}+\mathrm{K}-\mathrm{Cl} \\
/ \mathrm{Na}+ \\
\mathrm{k}+\mathrm{Cl}-\mathrm{Ca}\end{array}$ \\
\hline 1 & 0.01 & 0.05 & 1.68 & 0.01 & 0.47 & 143.00 & 0.00 & 0.00 & 0.03 & 0.00 & 0.00 & 1.68 & 0.05 & 0.48 & 0.99 & 277.27 & 0.01 & 0.09 \\
\hline 2 & 0.01 & 0.02 & 1.10 & 0.00 & 0.22 & 112.22 & 1.08 & 22.51 & 0.05 & 0.00 & 0.00 & 1.10 & 0.02 & 0.48 & 0.99 & 216.39 & 0.01 & 0.43 \\
\hline 3 & 0.01 & 0.09 & 1.89 & 0.01 & 0.22 & 143.93 & 1.06 & 11.77 & 0.05 & 0.00 & 0.00 & 1.89 & 0.09 & 0.48 & 0.99 & 279.21 & 0.01 & 0.25 \\
\hline 4 & 0.09 & 0.01 & 1.41 & 0.00 & 0.50 & 42.35 & 2.85 & 4.97 & 0.07 & 0.00 & 0.00 & 1.41 & 0.01 & 0.26 & 0.98 & 57.21 & 0.01 & -0.02 \\
\hline 5 & 0.00 & 0.00 & 0.00 & 0.00 & 0.41 & 151.79 & 1.06 & 20.03 & 0.03 & 0.00 & 0.00 & 0.00 & 1.00 & 0.48 & 0.99 & 294.71 & 0.00 & -0.11 \\
\hline
\end{tabular}


R. A. Il Akoachere et al.

\section{Continued}

\begin{tabular}{|c|c|c|c|c|c|c|c|c|c|c|c|c|c|c|c|c|c|c|}
\hline 6 & 0.00 & 0.00 & 0.00 & 0.00 & 0.11 & 0.00 & 0.00 & 0.00 & 0.08 & 0.00 & 0.00 & 0.00 & 1.00 & 0.49 & 0.99 & 297.27 & 0.00 & -0.83 \\
\hline 7 & 0.02 & 0.10 & 1.83 & 0.02 & 0.86 & 0.00 & 2.27 & 5.23 & 0.03 & 0.00 & 0.00 & 1.83 & 0.09 & 0.31 & 1.00 & 331.06 & 0.01 & -0.04 \\
\hline 8 & 0.07 & 0.06 & 1.27 & 0.01 & 0.23 & 25.04 & 0.00 & 4.91 & 0.07 & 0.00 & 0.00 & 1.27 & 0.05 & 0.42 & 0.96 & 43.13 & 0.01 & 0.18 \\
\hline 9 & 0.22 & 0.09 & 2.03 & 0.02 & 0.61 & 12.25 & 1.35 & 4.95 & 0.03 & 0.00 & 0.00 & 2.03 & 0.08 & 0.43 & 0.92 & 21.33 & 0.01 & 0.01 \\
\hline 10 & 0.12 & 0.04 & 1.01 & 0.02 & 0.61 & 13.01 & 0.00 & 0.00 & 0.03 & 0.00 & 0.00 & 1.01 & 0.04 & 0.39 & 0.93 & 21.46 & 0.01 & 0.27 \\
\hline 11 & 0.00 & 0.00 & 0.00 & 0.02 & 0.68 & 0.00 & 1.27 & 4.67 & 0.02 & 0.00 & 0.00 & 0.00 & 1.00 & 0.44 & 0.99 & 235.47 & 0.00 & -0.62 \\
\hline 12 & 0.02 & 0.07 & 1.64 & 0.02 & 0.54 & 0.00 & 1.27 & 4.80 & 0.03 & 0.00 & 0.00 & 1.64 & 0.06 & 0.44 & 0.99 & 212.52 & 0.01 & 0.10 \\
\hline 13 & 0.12 & 0.05 & 2.04 & 0.02 & 0.71 & 14.41 & 0.83 & 0.00 & 0.01 & 0.00 & 0.00 & 2.04 & 0.04 & 0.55 & 0.94 & 31.70 & 0.01 & 0.28 \\
\hline 14 & 0.25 & 0.08 & 1.69 & 0.04 & 1.56 & 0.00 & 1.85 & 0.00 & 0.01 & 0.00 & 0.00 & 1.69 & 0.08 & 0.35 & 0.92 & 18.91 & 0.01 & -0.02 \\
\hline 16 & 0.00 & 0.00 & 0.00 & 0.01 & 0.16 & 3.84 & 1.12 & 3.89 & 0.06 & 0.00 & 0.00 & 0.00 & 1.00 & 0.47 & 0.79 & 7.25 & 0.00 & -0.95 \\
\hline 17 & 0.00 & 0.00 & 0.00 & 0.00 & 0.00 & 0.00 & 2.81 & 0.00 & 0.06 & 0.00 & 0.00 & 0.00 & 1.00 & 0.26 & 0.99 & 95.00 & 0.00 & -0.56 \\
\hline 18 & 0.00 & 0.00 & 0.00 & 0.00 & 0.12 & 86.79 & 0.75 & 15.27 & 0.07 & 0.00 & 0.00 & 0.00 & 1.00 & 0.57 & 0.99 & 202.58 & 0.00 & -0.18 \\
\hline 19 & 0.00 & 0.00 & 0.00 & 0.00 & 0.10 & 71.89 & 0.65 & 13.61 & 0.07 & 0.00 & 0.00 & 0.00 & 1.00 & 0.61 & 0.99 & 183.00 & 0.00 & -0.23 \\
\hline 20 & 0.00 & 0.00 & 0.00 & 0.03 & 0.40 & 50.00 & 0.49 & 4.44 & 0.01 & 0.00 & 0.00 & 0.00 & 1.00 & 0.67 & 0.98 & 151.63 & 0.00 & -2.16 \\
\hline Min & 0.00 & 0.00 & 0.00 & 0.00 & 0.00 & 0.00 & 0.00 & 0.00 & 0.01 & 0.00 & 0.00 & 0.00 & 0.00 & 0.18 & 0.79 & 7.25 & 0.00 & -2.16 \\
\hline $\operatorname{Max}$ & 0.25 & 0.10 & 2.04 & 0.04 & 1.56 & 617.00 & 4.41 & 41.00 & 0.08 & 0.00 & 0.00 & 2.04 & 1.00 & 0.67 & 1.00 & 756.85 & 0.05 & 0.53 \\
\hline Mean & 0.05 & 0.03 & 0.89 & 0.01 & 0.47 & 74.38 & 1.26 & 8.10 & 0.04 & 0.00 & 0.00 & 0.89 & 0.43 & 0.44 & 0.97 & 186.70 & 0.01 & -0.18 \\
\hline Std. & 0.08 & 0.04 & 0.85 & 0.01 & 0.37 & 138.60 & 1.13 & 10.29 & 0.02 & 0.00 & 0.00 & 0.85 & 0.48 & 0.12 & 0.05 & 174.24 & 0.01 & 0.61 \\
\hline
\end{tabular}

(c)

\begin{tabular}{|c|c|c|c|c|}
\hline Ionic ratio & Wet & Dry & Comment & Interpretation \\
\hline $\mathrm{SO}_{4} / \mathrm{Cl}$ & $0.00-0.57$ & $0-0.25$ & high & Additional sources of $\mathrm{SO}_{4}$ from weathering of sulfates \\
\hline $\mathrm{Na} / \mathrm{Cl}$ & $0.00-0.07$ & $0-0.01$ & low & No Na-adsorption during freshening and a little silicate weathering \\
\hline $\mathrm{Mg} / \mathrm{Cl}$ & 0.00 to 0.73 & $0-2.04$ & high & Cation-exchange and silicate weathering of sandstones. \\
\hline $\mathrm{Na} / \mathrm{HCO}_{3}$ & $0.01-0.25$ & $0-0.004$ & high & Substantial weathering of Na-feldspar or other Na-silicates \\
\hline $\mathrm{Ca} / \mathrm{HCO}_{3}$ & $0.00-8.74$ & $0-1.56$ & high & Calc-carbonate dissolution or Calc-silicate weathering \\
\hline $\mathrm{Ca} / \mathrm{SO}_{4}$ & $1.53-21.97$ & $3.84-617$ & high & Gypsum dissolution present \\
\hline $\mathrm{Ca} / \mathrm{Mg}$ & $0.00-10.75$ & $0-4.41$ & high & Cation-exchange of weathering of silicate rocks. \\
\hline $\mathrm{Ca}+\mathrm{Mg} / \mathrm{Na}+\mathrm{K}$ & $0.00-10.65$ & $0.00-41.00$ & high & Carbonate weathering \\
\hline $\mathrm{HCO}_{3} / \Sigma$ Anions & $0.00-0.05$ & $0.01-0.08$ & high & Weathering reactions and input of dissolved species in recharge area \\
\hline $\mathrm{NO}_{3} / \Sigma$ Anions & $0.00-2.8 \mathrm{E}-6$ & $0.00-5.6 \mathrm{E}-6$ & very low & No anthropogenic contribution \\
\hline $\mathrm{SO}_{4} / \Sigma$ Anions & $0.00-0.01$ & $0.00-2.3 \mathrm{E}-3$ & very low & No oxidation of sulphides. \\
\hline $\mathrm{Mg} / \mathrm{Ca}$ & $0.09-1.28$ & $0.00-2.04$ & low & Weathering of Silicate rocks \\
\hline $\mathrm{Na} / \mathrm{Na}+\mathrm{Cl}$ & $0.00-1.00$ & $0.00-1.00$ & high & Sodium source other than halite-albite, ion exchange \\
\hline $\mathrm{Mg} / \mathrm{Ca}+\mathrm{Mg}$ & $0.09-0.56$ & $0.18-0.67$ & high & Dolomite dissolution, calcite precipitation or saltwater \\
\hline $\mathrm{Ca} / \mathrm{Ca}+\mathrm{SO}_{4}$ & $0.60-0.96$ & $0.79-1.00$ & high & Calcium source other than gypsum \\
\hline $\mathrm{Ca}+\mathrm{Mg} / \mathrm{SO}_{4}$ & $2.96-24.46$ & $7.25-756.85$ & & \\
\hline $\mathrm{Na}+\mathrm{K}-\mathrm{Cl} / \mathrm{Na}+\mathrm{K}-\mathrm{Cl}+\mathrm{Ca}$ & $-0.31-0.87$ & $-2.16-0.53$ & high & Plagioclase weathering unlikely \\
\hline $\mathrm{Cl} / \Sigma$ Anions & $0.00-0.14$ & $0.00-0.05$ & low & Rock weathering \\
\hline
\end{tabular}


12 of the $1866.7 \%$ ionic ratios calculated gave indices indicating weathering of geologic formations in the Mamfe basin as a source of solute concentration in the groundwater while nitrate ratio indicates no anthropogenic contribution and sulfate indices indicates no oxidation of sulfides. Ca is sourced from gypsum while $\mathrm{Na}$ is sourced from halite-albite and ion exchange. $\mathrm{Mg}$ is contributed by dolomite dissolution, calcite precipitation or saltwater. There is no plagioclase weathering. These high indices values are found in the following localities Lalla, Okoyong, Bachuo-Ntai, Bachuo-Akagbe and Egbekaw.

\section{Gibbs diagrams of groundwater in Mamfe}

The Gibbs diagrams were used. In the wet season 17 samples $85 \%$ plot in the rock-weathering dominance field and 3 samples $85 \%$ plot in the atmospheric precipitation dominance field. In the dry season 16 samples $80 \%$ plot in the rock-weathering dominance field and 4 samples $20 \%$ plot in atmospheric dominance field Figure 11. This indicates the mechanism contributing solute to groundwater in Mamfe is rock-weathering $80 \%$ - $85 \%$ followed by atmospheric precipitation $15 \%-20 \%$ in the dry and wet season respectively (Table 6).

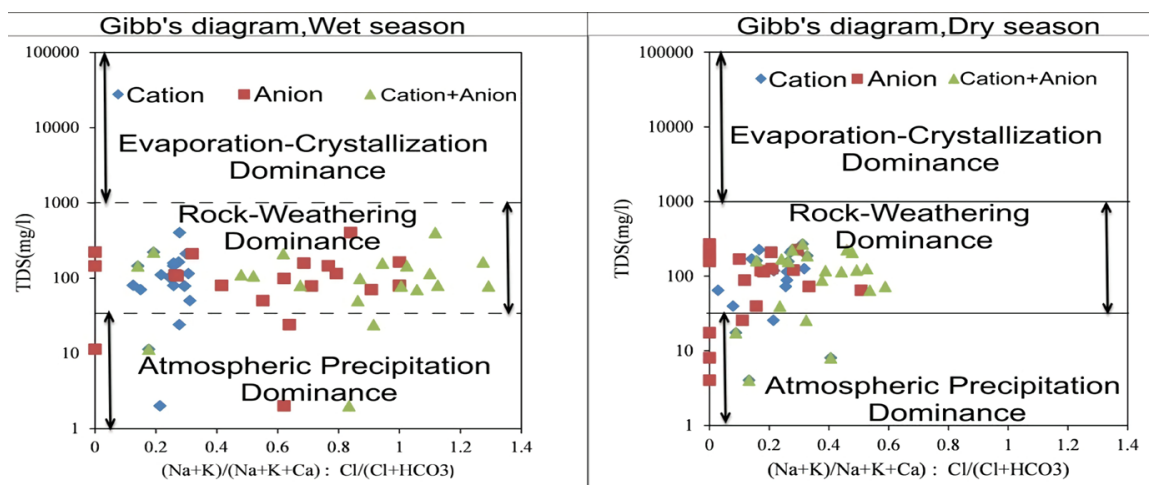

Figure 11. Gibbs diagrams for Mamfe groundwater (Gibbs, 1970): In the wet season 17 samples $85 \%$ cations and 17 samples $85 \%$ anions plot in the rock-weathering dominance field and 3 samples $15 \%$ cations and 3 samples $15 \%$ anions plot in atmospheric dominance field; In the dry season 16 samples $80 \%$ cations and 16 samples $80 \%$ anions plot in the rock-weathering dominance field and 4 samples $20 \%$ cations and 4 samples $20 \%$ anions plot in atmospheric dominance field; indicating the mechanism contributing groundwater constituents in the study area is rock-weathering and atmospheric precipitation.

Table 6. Gibbs Classification of groundwater in Mamfe and environs (Gibbs, 1970).

\begin{tabular}{|c|c|c|c|c|c|c|c|c|c|}
\hline \multirow{2}{*}{ Type } & \multirow{2}{*}{ Range } & \multicolumn{4}{|c|}{ Wet } & \multicolumn{4}{|c|}{ Dry } \\
\hline & & Cation & $\%$ & Anion & $\%$ & Cation & $\%$ & Anion & $\%$ \\
\hline Rock - Weathering dominance & $50-1000$ & 17 & 85 & 17 & 85 & 16 & 80 & 16 & 80 \\
\hline $\begin{array}{c}\text { Atmospheric Precipitation } \\
\text { dominance }\end{array}$ & $1-50$ & 3 & 15 & 3 & 15 & 4 & 10 & 4 & 20 \\
\hline
\end{tabular}

\section{Groundwater types}

The diamond field of piper diagram after Piper (1944) has further been divided into seven fields classifying water types and designated with alphabets from A to G, Langguth (1966). Using this classification, the water from the study area is dis- 
tinguished into the $\mathrm{A}, \mathrm{B}$, and $\mathrm{C}$ categories. The $\mathrm{D}, \mathrm{E}, \mathrm{F}$, and $\mathrm{G}$ water types are absent. In the rainy season; Category A, 3 samples 15\%; characterized by normal earth alkaline water with prevailing bicarbonate. Category B, 3 samples 15\% are characterized by normal earth alkaline water with prevailing sulfate or chloride and Category C, 14 samples 70\% Figure 12 are characterized by alkaline earth water with increased portions of alkalis with prevailing bicarbonate. In the dry season; Category A, 14 samples 70\%, Category B, 5 samples 25\% Category C, 1 sample 5\%. The dominant water types are Category C 70\%; Normal earth alkaline water; prevailing $\mathrm{SO}_{4}^{2-}$ or $\mathrm{Cl}^{-}$in the wet season and Category A 70\%; Normal earth alkaline water; prevailing $\mathrm{NO}_{3}^{-}$in the dry season. The water types in Mamfe groundwater are $\mathrm{Ca}-\mathrm{SO}_{4}$ and $\mathrm{Mg}-\mathrm{HCO}_{3}$ for both seasons Table 7.

\section{Piper's Hydrogeochemical facies}

From the Piper's diagrams, field I: Ca- $\mathrm{Mg}-\mathrm{Cl}^{-}-\mathrm{SO}_{4}$ hydrogeochemical facies has 15 samples $75 \%$ in the rainy and 2 samples $10 \%$ in the dry season demonstrating the dominance of alkaline earths over alkali $\mathrm{Ca}+\mathrm{Mg}>\mathrm{Na}+\mathrm{K}$ and strong acidic anions over weak acidic anions. Field IV, Ca-Mg- $-\mathrm{HCO}_{3}$ hydrogeochemical facies has 5 samples $25 \%$ in the rainy and 18 samples $90 \%$ in the dry season Table 8 signifying the dominance of alkaline earths over alkali and weak acidic anions over strong acidic anions. This facies is characteristic of freshly recharged groundwater that has equilibrated with $\mathrm{CO}_{2}$ and soluble carbonate minerals under an open system conditions in the vadose zone typical of shallow groundwater flow systems in crystalline phreatic aquifers.

No samples plotted on field II and field III. The dominance of Ca-Mg- $\mathrm{HCO}_{3}$ hydrogeochemical facies in this area could be due to dissolution of gases and minerals, particularly $\mathrm{CO}_{2}$ and $\mathrm{CO}_{2}$-related compounds from the atmosphere
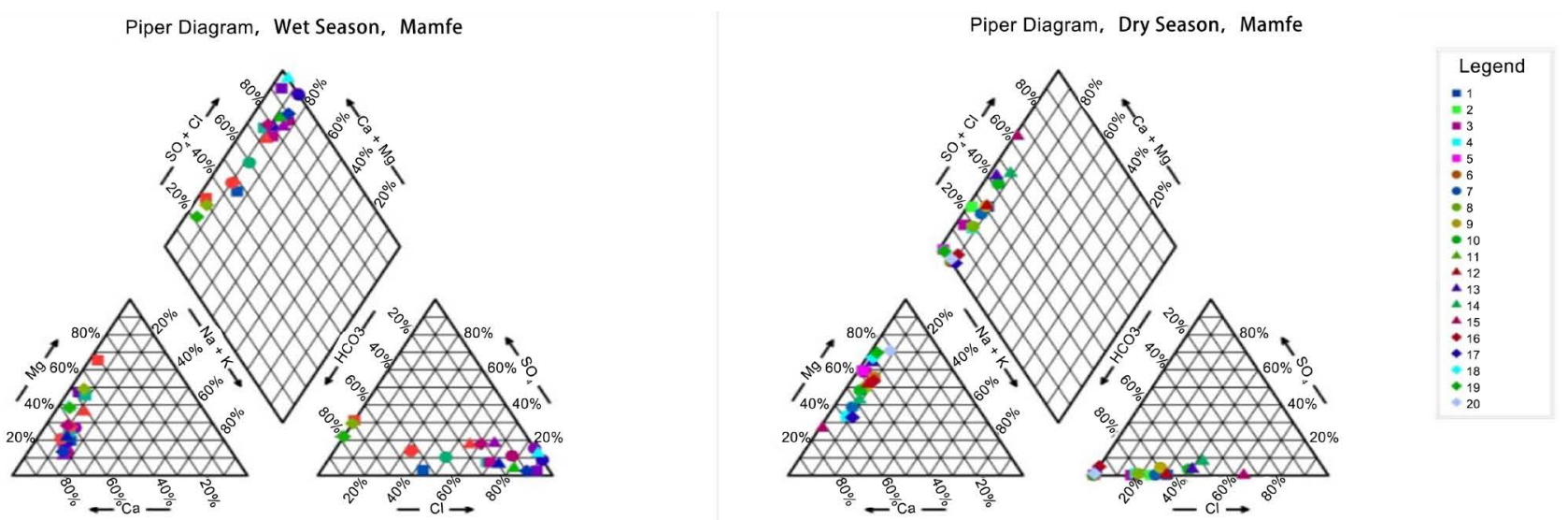

Figure 12. Piper's diagram for Mamfe groundwater samples; during rainy season in Category A, 3 samples $15 \%$; characterized by normal earth alkaline water with prevailing bicarbonate. Category B, 3 samples $15 \%$ are characterized by normal earth alkaline water with prevailing sulfate or chloride and Category C, 14 samples $70 \%$ are characterized by alkaline earth water with increased portions of alkalis with prevailing bicarbonate. In the dry season; Category A, 14 samples $70 \%$, Category B, 5 samples $25 \%$ and Category C, 1 sample 5\% are characterized by alkaline earth water with increased portions of alkalis with prevailing bicarbonate. Diamond field I: Ca-Mg-Cl-SO ${ }_{4}$ hydrogeochemical facies has 15 samples $75 \%$ in the rainy and 2 samples $10 \%$ in the dry season. Field IV, Ca- $\mathrm{Mg}-\mathrm{HCO}_{3}$ hydrogeochemical facies has 5 samples $25 \%$ in the rainy and 18 samples $90 \%$ in the dry season. No samples plotted on field II and field III in both seasons. Langguth (1966). 
Table 7. Classification of Mamfe groundwater based on Piper diagram (Langguth, 1966) to depict water types.

\begin{tabular}{cccccc}
\hline & & \multicolumn{2}{c}{ Wet } & \multicolumn{3}{c}{ Dry } \\
\hline Class & Description of Water Types & No & $\%$ & No & $\%$ \\
A & Normal earth alkaline water with prevailing bicarbonate & 3 & 15 & 14 & 70 \\
B & Normal earth alkaline water with prevailing bicarbonate and sulfate & & & & \\
or chloride & 3 & 15 & 5 & 25 \\
C & Normal earth alkaline water with prevailing Sulfate or Chloride & 14 & 70 & 1 & 5 \\
& Cations field & & & & \\
1 & Calcium rich & 18 & 90 & 6 & 30 \\
2 & Magnesium rich & 2 & 10 & 14 & 70 \\
& Anion Field & & & & \\
4 & Bicarbonate rich & 6 & 30 & 19 & 95 \\
5 & Chloride rich & 14 & 70 & 1 & 5 \\
\hline
\end{tabular}

Table 8. Classification of water based on Piper diagram (Langguth, 1966).

\begin{tabular}{cccccc}
\hline Field & Hydrogeochemical facies & Wet & $\%$ & Dry & $\%$ \\
\hline Field I & Ca-Mg-Cl-SO & 15 & 75 & 2 & 10 \\
Field IV & Ca-Mg- $\mathrm{HCO}_{3}$ & 5 & 25 & 18 & 90 \\
\hline
\end{tabular}

dissolved in precipitation and during groundwater infiltration through the vadose zone.

\section{Durov diagram}

Based on the classification of Lloyd and Heathcoat (1985): Six classes of processes occur in the rainy season; Class 1 recharging waters: 10 samples 50\%; Class 2 ion exchange water: 5 samples 15\%; Class 3 ion exchange water: 1 samples 5\%; Class 5 simple dissolution or mixing: 1 samples 5\%; Class 6 probable mixing or uncommon dissolution influences: 1 sample respectively $5 \%$ and Class 72 samples respectively 10\%; $\mathrm{Cl}$ and $\mathrm{Na}$ dominant is frequently encountered, Otherwise the water may result from reverse ion exchange of $\mathrm{Na}-\mathrm{Cl}$ waters $\mathrm{Ta}$ ble 9. Five classes of processes occur in the dry season: Class 2 ion exchange water: 1 sample 5\%; Class 3 ion exchange water: 3 samples 15\%; Class 5 simple dissolution or mixing: 1 sample 5\%; Class 6 probable mixing or uncommon dissolution influences: 11 samples 55\% respectively Figure 13. There are no Classes 4 and 9 in the rainy season and no Classes 1, 7 and 9 in the dry season samples in groundwater from Mamfe. In the rainy season, fresh recently recharging water exchanges ions with the matrix of the formation, while simple dissolution or mixing also goes on between the recently recharging and the existing groundwater in the formation. In the dry season, recharged groundwater having spent more time in the formation continues to exchange ions to a lesser extent with the matrix of the formation while increasingly; simple dissolution or mixing also goes on between the recently recharged groundwater and the pre-existing groundwater in the formation. 

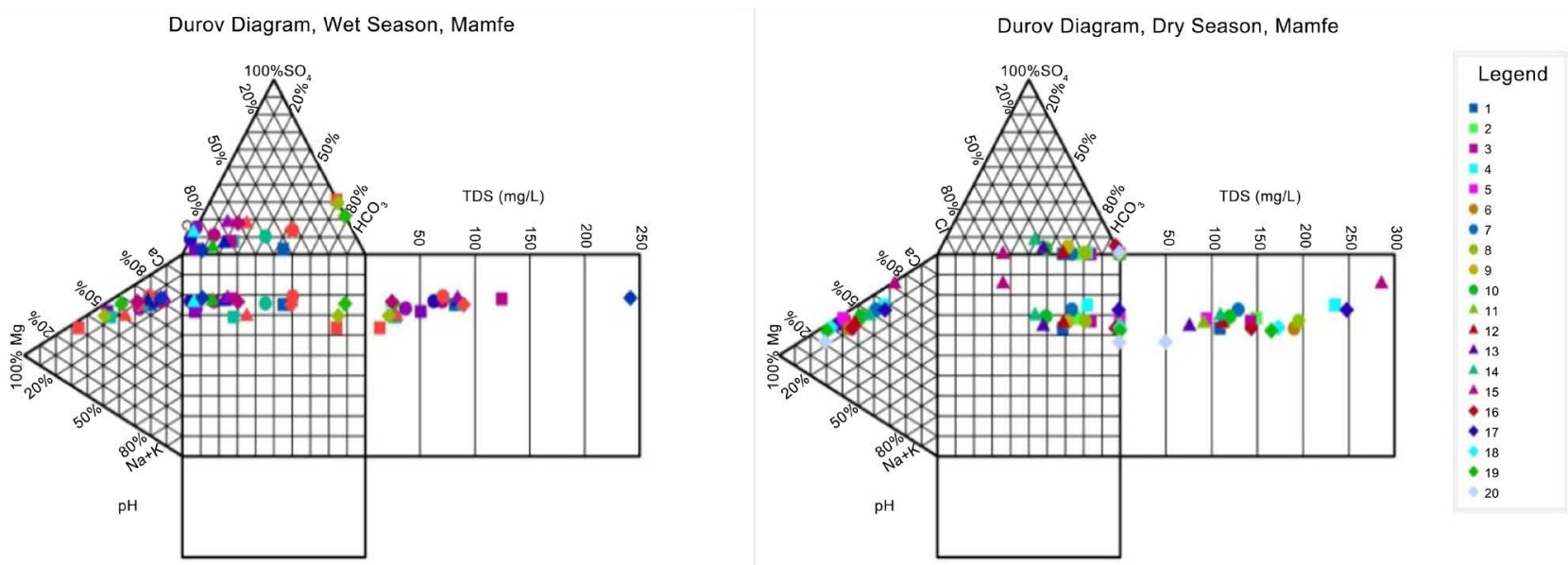

Figure 13. From Durov diagrams: Six classes of processes occur in the wet season: Class 1 recharging waters: 10 samples 50\%; Class 2 ion exchange water: 5 samples 25\%; Class 3 ion exchange water: 1 samples 5\%; Class 51 sample 5\% simple dissolution or mixing: Class 61 sample $5 \%$ probable mixing or uncommon dissolution influences: and Class 72 sample respectively $10 \%$; $\mathrm{Cl}$ and $\mathrm{Na}$ dominant is frequently encountered. Otherwise the water may result from reverse ion exchange of Na-Cl waters. Five classes of processes occur in the dry season: Class 2 ion exchange water: 1 sample 5\%; Class 3 ion exchange water: 3 samples 15\%; Class 5 simple dissolution or mixing: 5 samples 25\%; Class 6 probable mixing or uncommon dissolution influences: 11 samples $55 \%$ respectively. Durov's 1948; Lloyd \& Heathcoat (1985).

Table 9. Classification of Water based on Durov diagram (Lloyd \& Heathcoat, 1985).

\begin{tabular}{|c|c|c|c|c|c|}
\hline \multirow[b]{2}{*}{ SN } & \multirow[b]{2}{*}{ Description of Water Types } & \multicolumn{2}{|c|}{ Wet } & \multicolumn{2}{|c|}{ Dry } \\
\hline & & No & $\%$ & No & $\%$ \\
\hline 1 & $\begin{array}{l}\mathrm{HCO}_{3} \text { and } \mathrm{Ca} \text { dominant, frequently indicates recharging waters in } \\
\text { limestone, sandstone, and many other aquifers }\end{array}$ & 10 & 50 & 0 & 0 \\
\hline 2 & $\begin{array}{l}\text { This water type is dominated by } \mathrm{Ca} \text { and } \mathrm{HCO}_{3} \text { ions. Association with } \\
\text { dolomite is presumed if } \mathrm{Mg} \text { is significant. However, those samples in } \\
\text { which } \mathrm{Na} \text { is significant, an important ion exchanged is presumed }\end{array}$ & 5 & 25 & 1 & 5 \\
\hline 3 & $\begin{array}{l}\mathrm{HCO}_{3} \text { and } \mathrm{Na} \text { are dominant, normally indicates ion exchanged water, } \\
\text { although the generation of } \mathrm{CO}_{2} \text { at depth can produce } \mathrm{HCO}_{3} \text { where } \mathrm{Na} \text { is } \\
\text { dominant under certain circumstances }\end{array}$ & 1 & 5 & 3 & 15 \\
\hline 5 & $\begin{array}{l}\text { No dominant anion or cation, indicates water exhibiting simple } \\
\text { dissolution or mixing }\end{array}$ & 1 & 5 & 5 & 25 \\
\hline 6 & $\begin{array}{l}\mathrm{SO}_{4} \text { dominant or anion discriminate and } \mathrm{Na} \text { dominant; is water type that } \\
\text { is not frequently encountered and indicates probable mixing or } \\
\text { uncommon dissolution influences. }\end{array}$ & 1 & 5 & 11 & 55 \\
\hline 7 & $\begin{array}{l}\mathrm{Cl} \text { and } \mathrm{Na} \text { dominant are frequently encountered or the water may have } \\
\text { resulted from reverse ion exchange of } \mathrm{Na}-\mathrm{Cl} \text { waters }\end{array}$ & 2 & 10 & 0 & 0 \\
\hline
\end{tabular}

\subsection{Water Quality}

\section{Domestic Water Quality}

Ionic content of water in the study area was used to evaluate groundwater suitability for domestic use: The recommended values are of the WHO (2017) guidelines. The quality standards for drinking water have been specified by the World Health Organization (WHO) 2017. The suitability of groundwater in the study area based on WQI and total hardness $\mathrm{H}_{\mathrm{T}}$ are discussed below.

\section{Water quality index (WQI)}


The WHO (2017) permissible values of ions present in the groundwater have been used to calculate WQI values, Asadi et al. (2007). Water Quality Index WQI considered the most effective tool to convey the water quality information in the simplest form to the public (Babaei, 2011). The WQI values range from 0 70.79 in the wet season and $13.2-276.6$ in the dry. $80 \%$ of the water samples in the wet season can be considered suitable for domestic and other utilitarian purposes as they belong to excellent to good water quality classes Table 10. Remaining $20 \%$ of the samples are said to be unfit for consumption as they belong to poor class Figure 14 whereas $20 \%$ of the water samples in the dry season can be considered suitable for domestic and other utilitarian purposes as they belong to excellent to good water quality classes. Remaining $80 \%$ of the samples are said to be unfit for consumption as they belong to poor, very poor and unsuitable classes Table 10.

\section{Total hardness (TH)}

The total hardness of groundwater samples range from $20.63-244.87 \mathrm{mg} / \mathrm{L}$ in the wet season and $86.66-338.47 \mathrm{mg} / \mathrm{L}$ in the dry season Figure $15.80 \%$ of groundwater in the study area can be classified as soft, $10 \%$ fall into the moderately hard category, and just $10 \%$ in the wet season is hard water that may be a potential health risk factor whereas in the dry season $0 \%$ of groundwaters in the study area can be classified as soft, $65 \%$ fall into the moderately hard category, $30 \%$ is hard water and just $5 \%$ is very hard Table 11 .

Table 10. WQI classification indicating the suitability of water for drinking.

\begin{tabular}{cccccc}
\hline Index & Quality & WQI-wet & $\%$ & WQI-dry & $\%$ \\
\hline $0-25$ & Excellent & 10 & 50 & 1 & 5 \\
$26-50$ & Good & 6 & 30 & 3 & 15 \\
$51-75$ & Poor & 4 & 20 & 2 & 10 \\
$76-100$ & Very poor & 0 & 0 & 0 & 0 \\
$>100$ & Unsuitable & 0 & 0 & 14 & 70
\end{tabular}

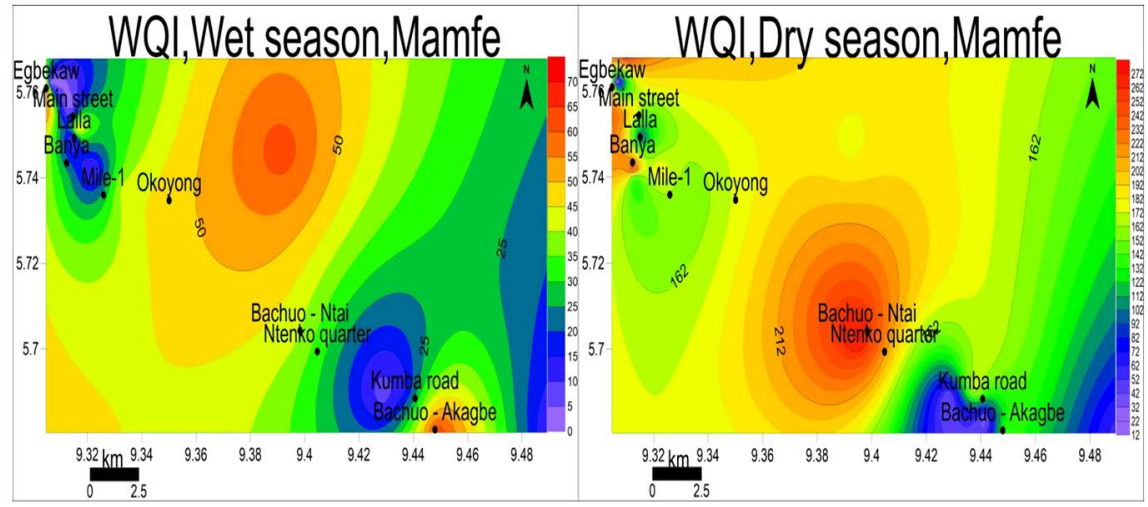

Figure 14. Spatial variation in water quality index (WQI) values of dug well water in Mamfe during wet season and dry seasons. Note increase in WQI values during the dry season and decrease WQI values in the wet season. 
Table 11. Hardness classification of groundwater of the study area (Sawyer \& McCarty, 1967).

\begin{tabular}{cccccc}
\hline & & \multicolumn{2}{c}{ Wet } & \multicolumn{2}{c}{ Dry } \\
\hline Hardness $(\mathrm{mg} / \mathrm{L})$ & Classification & No & $\%$ & No & $\%$ \\
\hline $0-75$ & Soft & 16 & 80 & 0 & 0 \\
$76-150$ & Moderately Hard & 2 & 10 & 13 & 65 \\
$151-300$ & Hard & 2 & 10 & 6 & 30 \\
$>300$ & Very Hard & 0 & 0 & 1 & 5 \\
\hline
\end{tabular}

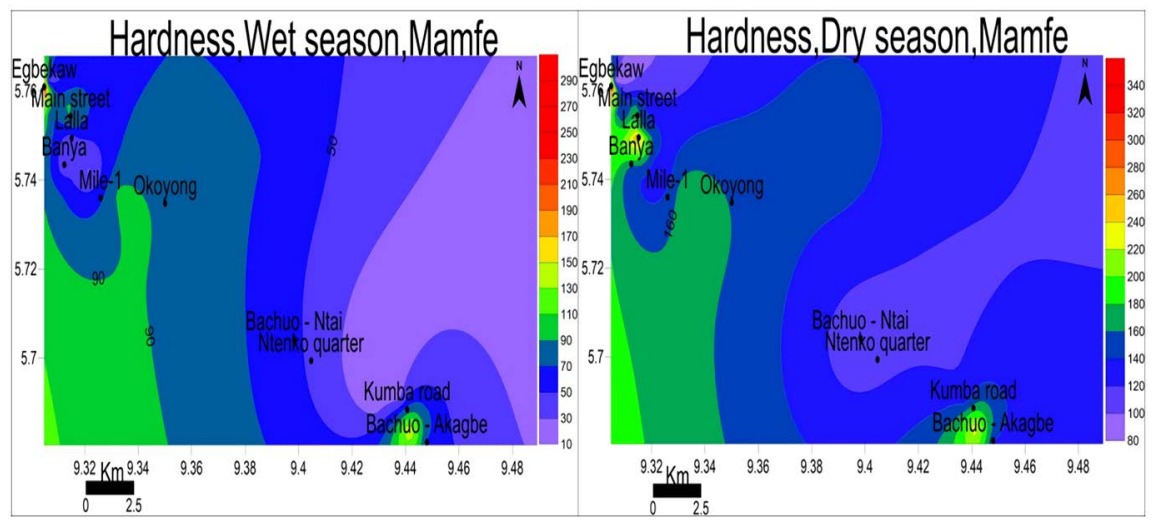

Figure 15. Spatial variation of dug well water total hardness in study area during wet and dry season; $80 \%$ of groundwaters in the study area can be classified as soft, $10 \%$ fall into the moderately hard category, and just $10 \%$ in the wet season is hard water season; $0 \%$ of groundwaters in the study area can be classified as soft, $65 \%$ fall into the moderately hard category, $30 \%$ is hard water and just $5 \%$ is very hard in the dry season.

\subsection{Water Quality for Irrigational Use}

The important parameters which determine the irrigation water quality of the study area are discussed below;

\section{Sodium percent}

Sodium percent values range from 3.64 - 16.59 in wet season and $1.22-10.97$ dry season. Sodium along with carbonate forms alkaline soil; while sodium with chloride forms saline soil; both of these are not suitable for the growth of plants (Pandian \& Shankar 2007). The quality classifications of irrigation water based on the values of sodium percentage as proposed by Wilcox (1955) suggest that the groundwater of the study area is good to permissible category both in the wet and dry season Figure 16 indicating the water is suitable for irrigation (Figure 17).

\section{Sodium adsorption ratio}

SAR values range from $0.005-0.075$ in rainy season and $0.01-0.14$ during dry season. Based on the SAR values, all samples have low sodium hazard and on plotting over the USSL Salinity diagram (USSL 1954) Figure 18. All the 20 groundwater samples fall in the $\mathrm{S}_{1}-\mathrm{C}_{0-3}$ classes Table 12 for both rainy season and dry season considered suitable for irrigation (Figure 19). 


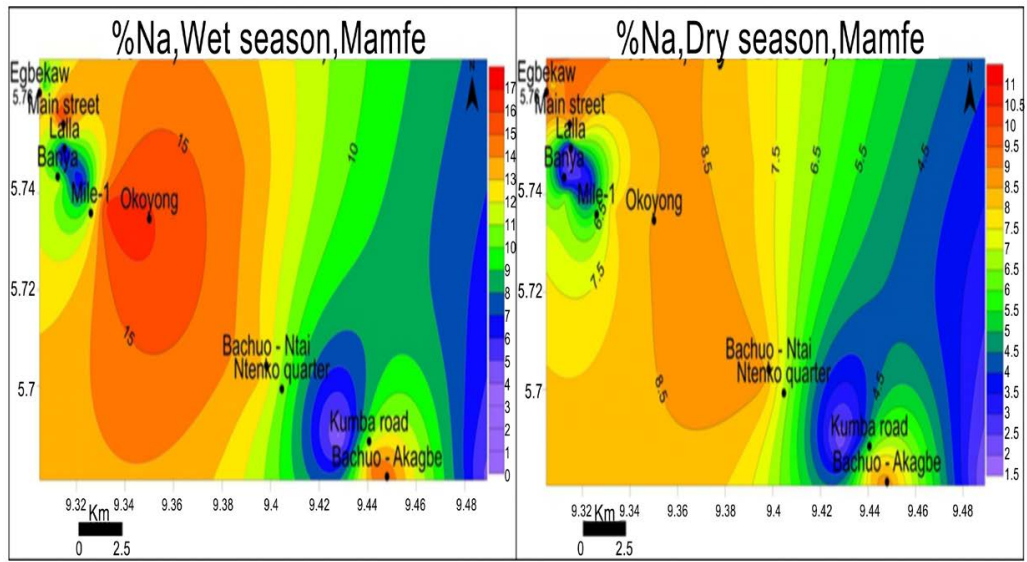

Figure 16. Spatial variation of dug well water sodium percentage $\% \mathrm{Na}$ in Mamfe during wet season and dry season. Note decrease in $\% \mathrm{Na}$ values in the dry season while in the wet the $\% \mathrm{Na}$ values increases.

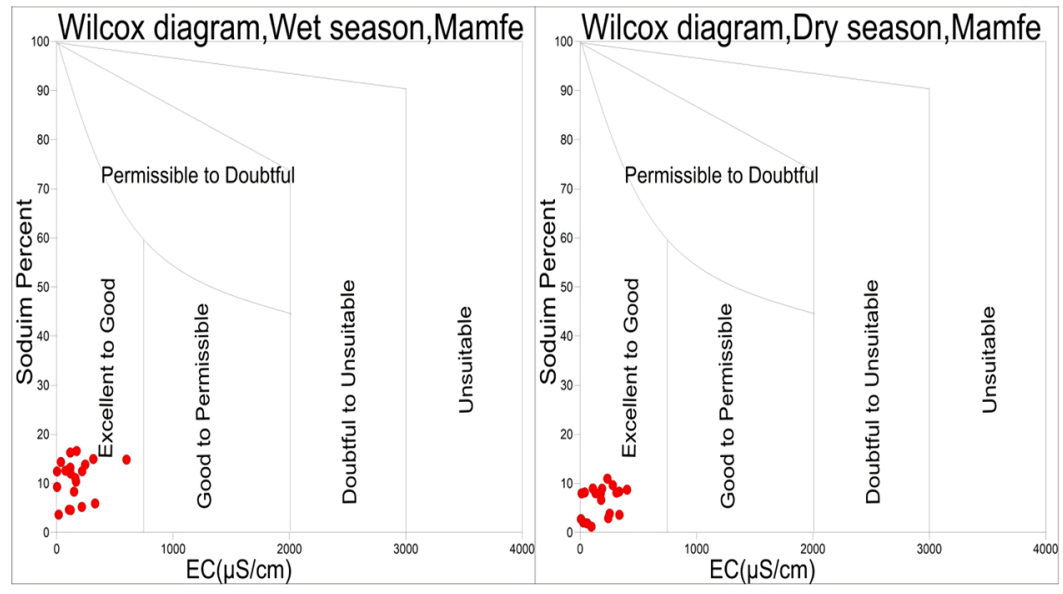

Figure 17. Wilcox diagram showing groundwater suitability for irrigation with all the water samples plotting in excellent to good fields in both wet and dry seasons indicating that the water is suitable for irrigation in both seasons.

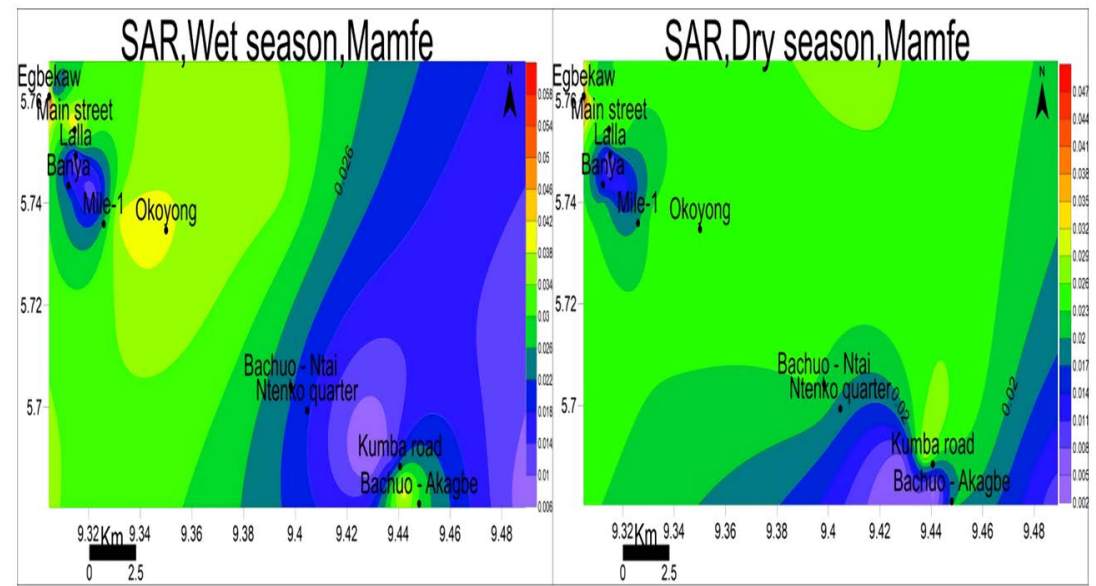

Figure 18. Spatial variation of dug well water Sodium absorption ratio SAR in Mamfe during wet season and dry seasons. Note increase in SAR values during the wet season while in the dry season the SAR values decreases. 
Table 12. Water quality classification based on EC of Mamfe groundwater.

\begin{tabular}{ccccccc}
\hline $\begin{array}{c}\text { Hazard } \\
\text { Class }\end{array}$ & EC $(\mu \mathrm{S} / \mathrm{cm})$ & Quality & No & $\%$ & No & $\%$ \\
\hline C0 & $0-100$ & Excellent & 4 & 20 & 6 & 30 \\
C1 & $101-250$ & Very Good & 13 & 65 & 8 & 40 \\
C2 & $251-750$ & Good & 3 & 15 & 6 & 30 \\
\hline
\end{tabular}

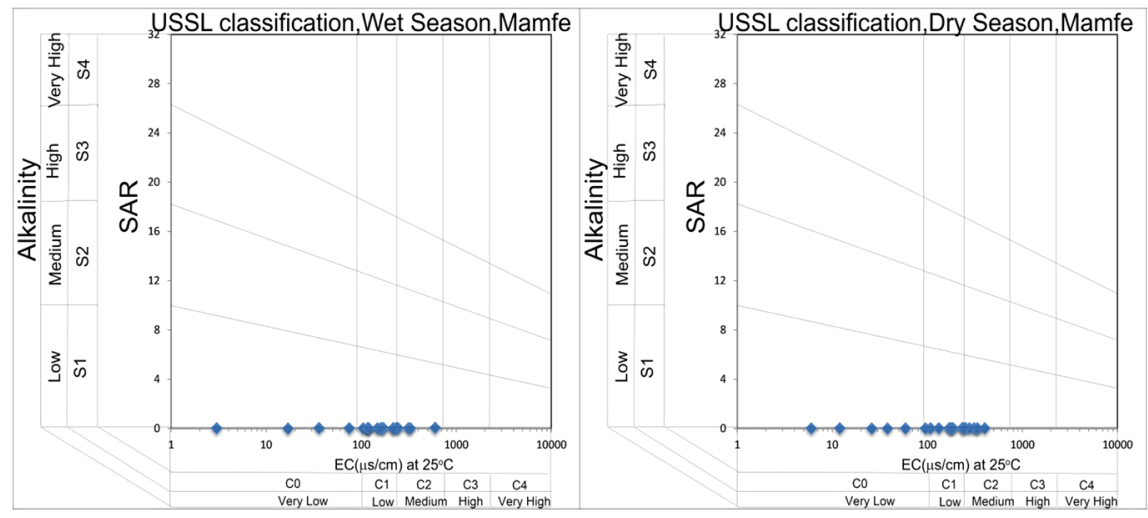

Figure 19. Residual Salinity Hazard classification, Mamfe; The $\mathrm{S}_{1} \mathrm{C}_{0}, \mathrm{~S}_{1} \mathrm{C}_{1}$, and $\mathrm{S}_{1} \mathrm{C}_{2}$ make up the excellent, very good and good classes respectively. In the Wet season 4 samples $20 \%$ plotted in the excellent class, 13 samples $65 \%$ plotted in the very good class and 3 samples $30 \%$ potted in the good class whereas during the dry season 6 samples $30 \%$ plotted in the excellent class and 8 samples $40 \%$ plotted in the good field and 6 samples $30 \%$ potted in the good class.

\section{Permeability index}

The PI values range 0.89 - 68.63 in the wet season and $18.75-73.35$ in the dry season Figure 20. The classification of irrigation waters has been attempted on the basis of permeability Index, as suggested by Doneen (1962). The groundwater samples of the study area fall in class-I and II as per Doneen chart, the groundwater samples of the study area are of good quality for irrigation. The increased percentage of groundwater samples under class-I is due to dilution and subsequently the lower values of permeability index (Figure 21).

\section{Magnesium Adsorption Ratio}

Magnesium Ratio adsorption values range from 13.3 - 67.88 in the wet season and $27.02-77.01$ in the dry season Figure 22. Magnesium Ratio adsorption less than $50 \%$ it is considered as suitable for irrigation purpose. In the study area, $90 \%$ of the samples are suitable for irrigation during the wet season whereas $25 \%$ of the samples are suitable for irrigation during the dry season.

\section{Residual Sodium Carbonate}

The RSC values -4.59 to -0.33 in the wet season and $-5.13-0.3 \mathrm{mg} / \mathrm{L}$ in the dry season Figure 23. RSC values $<1.25 \mathrm{mg} / \mathrm{L}$ are considered as safe for irrigation while those from $1.25 \mathrm{mg} / \mathrm{L}$ to $2.5 \mathrm{mg} / \mathrm{L}$ are marginally suitable for irrigation. If RSC values are $>2.5$ the groundwater is unsuitable for irrigation (Eaton, 1950; Richards, 1954). All the RSC values are $<1.25$ in the study area thus rendering the water suitable for irrigation in both seasons. 


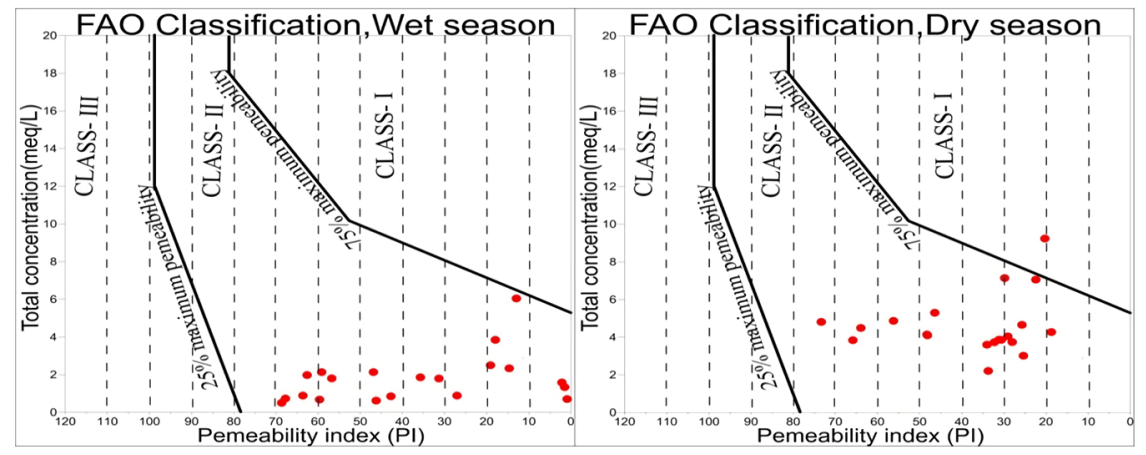

Figure 20. FAO classification of groundwater for irrigation indicating that the water is suitable for irrigation in the dry season as it is observed that the samples plot in class I and class II field indicating that the water is suitable for both seasons.

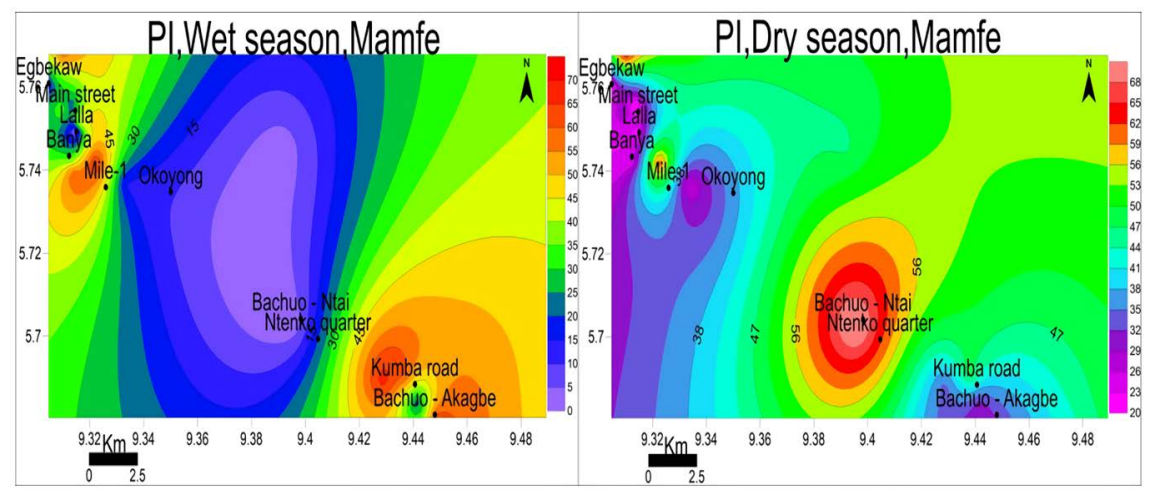

Figure 21. Spatial variation of permeability index in Mamfe; (a) Wet season (b) dry season; Permeability index is highest during the dry season at Bachuo-Akagbe and Ntenko quarter and lowest during the wet season at Bachuo-Ntai and Okoyong.

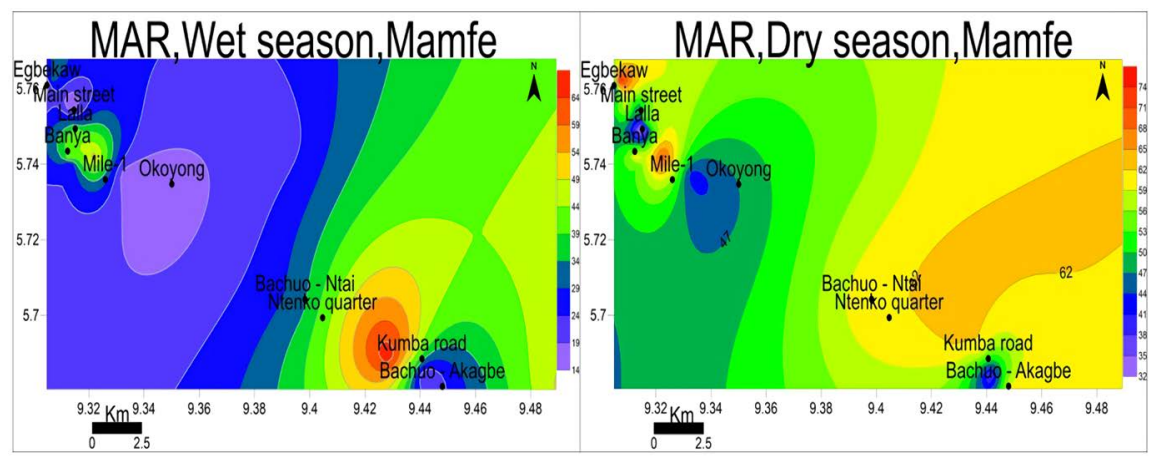

Figure 22. Spatial variation of dug well water Magnesium adsorption ratio in the study area during wet season and dry season. Note decrease in MAR values during the wet season while in the dry seasons the MAR values increases at Egbekaw, Bachuo-Ntai, Kumba road, Mile-1 and Ntenko quarter.

\section{Kelly's Ratio (KR)}

$\mathrm{KR}<1$ is considered suitable for irrigation and $\mathrm{KR}>1$ is unsuitable. During rainy season, $\mathrm{KR}$ values vary between $0.01-0.02$ and during the dry season the values vary between 0.00 - 0.02 Figure 24. All groundwater samples in Mamfe are suitable for irrigation for both seasons. 


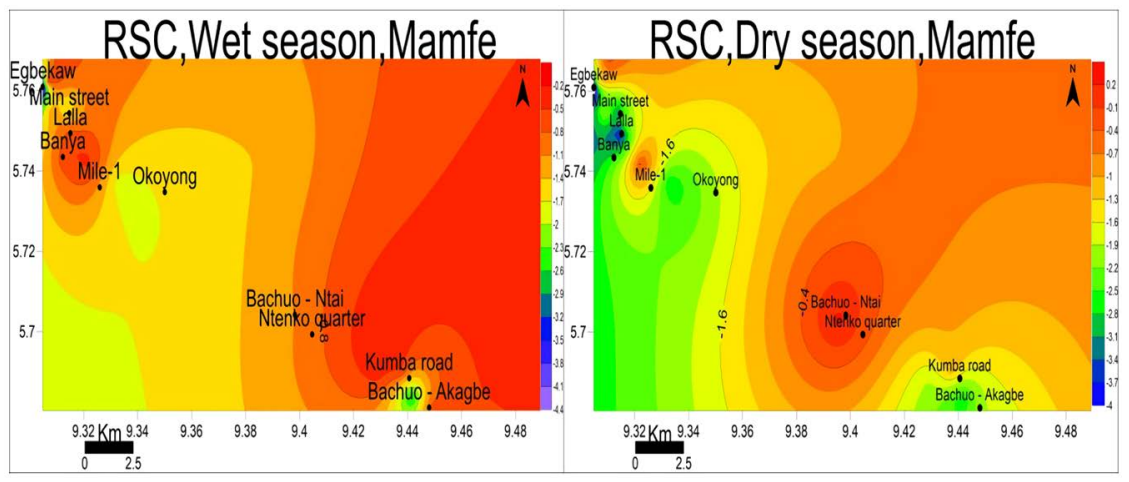

Figure 23. Spatial variation of dug well water residual sodium carbonate in the study area during wet season and dry season. Note decrease in RSC values during the wet season while in the dry seasons the RSC values increases.

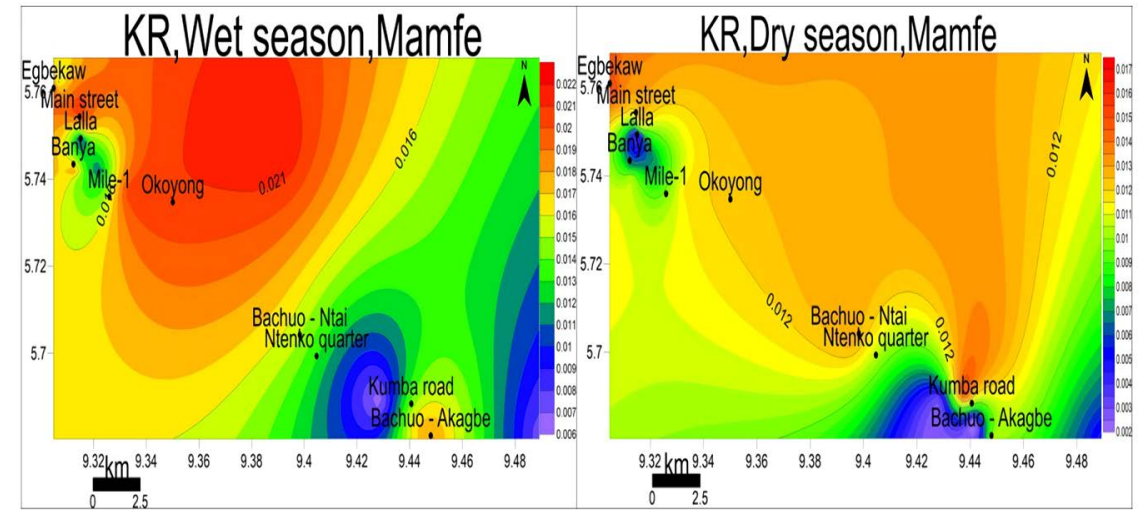

Figure 24. Spatial variation of dug well water Kelly's ratio in the study area during wet season and dry season. Note decrease in KR values during the wet season and dry seasons.

\section{Discussion}

Temperature, $\mathrm{pH}, \mathrm{EC}$ and TDS vary with seasons as such the groundwater is in hydraulic connectivity with the atmosphere indicative of a phreatic aquifer. All major ions fell below WHO (2017) acceptable limits for both seasons. From ionic ratios there are additional sources of $\mathrm{SO}_{4}$, silicate weathering possibly of the sandstones, conglomerates and other rocks in this area. Weathering of Na-feldspar or other $\mathrm{Na}$-silicates and $\mathrm{Ca}$-carbonate dissolution or $\mathrm{Ca}$-silicate weathering. $\mathrm{Ca}$ tion-exchange of the silicate rocks with the groundwater. Ironic ratio values for nitrate and sulfate are very low as such there are no anthropogenic contribution and no oxidation of sulphides. Solutes from weathering reactions and inputs of dissolved species in precipitation get into the aquifer indicating a recharge zone. From Gibbs diagram there is the dominance of rock-weathering and atmospheric precipitation dominance. From Durov diagrams the processes involve are ion exchange, dissolution and mixing. From the Piper's diagrams, the dominant hydrogeochemical facies are $\mathrm{Ca}-\mathrm{Mg}-\mathrm{Cl}-\mathrm{SO}_{4}$ and $\mathrm{Ca}-\mathrm{Mg}-\mathrm{HCO}_{3}$ for both seasons. This facies is characteristic of freshly recharged groundwater that has equilibrated with $\mathrm{CO}_{2}$ and soluble carbonate minerals under an open system conditions in the vadose zone typical of shallow groundwater flow systems in crystal- 
line phreatic aquifers.

From the above groundwater synthesis, WQI values indicate that $80 \%$ of the groundwater in the wet season can be considered suitable for domestic use, 20\% are classified to be unfit for consumption whereas in the dry season $20 \%$ are suitable for domestic purposes and $80 \%$ are unfit for consumption as they belong to poor, very poor and unsuitable classes. Therefore the water in Mamfe is more suitable for domestic purposes in the wet season than in the dry season similar to work done in Kumba, by Akoachere et al. (2018).

The values of SAR, PI, \% Na, KR and WQI, RSC and Wilcox diagram indicate that most groundwater in the study area are suitable for irrigation purposes but of MAR with values higher in Mile-1, Kumba road, Egbekaw, and Bachuo-Ntai rendering the water unfit for irrigation.

Since there exist little hydrogeological or hydrological work in this part of the basin, there is little to compare the results here-in, this being the first study that sheds light into these unexplored aspects of the basin.

\section{Conclusion}

The geogenic input to groundwater is the weathering of rocks possibly of the granites, gneisses, sandstones, conglomerates, shales and other rocks in this area.

Groundwater ionic content was as a result of ion exchange from rock-weathering of the aquiferous formations in the area, dissolution and recharge from precipitation.

Water types are: $\mathrm{CaSO}_{4}$ and $\mathrm{MgHCO}_{3}$ in both seasons. Precipitation recharge, ion-exchange and simple dissolution are the processes determining groundwater character.

Hydrogeochemical facies are $\mathrm{Ca}-\mathrm{Mg}-\mathrm{Cl}-\mathrm{SO}_{4}$ and $\mathrm{Ca}-\mathrm{Mg}-\mathrm{HCO}_{3}$.

All major ions concentrations are below WHO acceptable guidelines for both seasons.

Water quality indices: SAR, PI, \%Na, KR and WQI, RSC and Wilcox diagram indicate that water in the study area is irrigation suitability assessment but of MAR were the values that are higher in Mile-1, Kumba road, Egbekaw, and Bachuo-Ntai rendering the water unfit for irrigation.

There is a need for more studies in order to determine the aquifer extent: lateral and vertical, aquifer boundaries, aquifer hydraulic parameters (permeability, transmissivity and storage capacity), the biological and organic water quality, necessary tools for a good management of this important resource in the aquiferous formations in Mamfe. These more detailed studies will throw more light on the groundwater capacity and residence time in the different aquiferous formations in the Mamfe Basin.

\section{Funding}

This research did not receive any specific grant from funding agencies in the public, commercial, or not-for-profit sectors. 


\section{Conflicts of Interest}

None.

\section{References}

Akoachere, R. A., Ngwese, Y. M., Egbe, S. E., Eyong, T. A., Edimo, S. N., \& Tambe, D. B. (2018). Groundwater Monitoring in the Gneisso-Basaltic Fractured Rock Aquiferous Formations of Kumba, Southwest Region Cameroon: Seasonal Variations in the Aqueous Geochemistry and Water Quality. Journal of Geoscience and Environment Protection, 6, 18-50.

APHA American Public Health Association (1995). Standard Methods for Examination of Water and Waste Water. Washington DC: American Public Health Association, American Water Works Association and Water Pollution Control Federation.

Asadi, J. J., Vuppala, P., \& Reddy, M. A. (2007). Remote Sensing and GIS Techniques for Evaluation of Groundwater Quality in Municipal Corporation of Hyderabad (Zone-V), India. International Journal of Environmental Research and Public Health, 4, 45-52. https://doi.org/10.3390/ijerph2007010008

Babiker, I. S., Mohamed, M. A. A., \& Hiyama, T. (2007). Assessing Groundwater Quality Using GIS. Water Resources Management, 21, 699-715. https://doi.org/10.1007/s11269-006-9059-6

Bear, J. (1979). Hydraulics of Groundwater. New York: McGraw-Hill International Book.

Doneen, L. D. (1962). The Influence of Crop and Soil on Percolating Water. Proceedings of the 1961 Biennial Conference on Groundwater Recharge, 156-163.

Dumort, J. C. (1968). Reconnaissance Geologic Map of Douala-West (1:500000). and EXplanatory Notes. Federal Republic of Cameroon, Directorate of Mines and Geology Cameroon, 69.

Durov, S. A. (1948). Classification of Natural Waters and Graphical Representation of Their Composition. Doklady Akademii Nauk SSSR, 59, 87-90.

Eaton, E. M. (1950). Significance of Carbonate in Irrigation Water. Soil Science, 69, 123-134. https://doi.org/10.1097/00010694-195002000-00004

Eyong, J. T., Wignall, P., Fantong, W. Y., Best, J., \& Hell, J. V. (2013). Paragenetic Sequences of Carbonate Rocks and Sulphide Minerals of the Mamfe Basin (Cameroon): Indicators of Paleo-Fluids, Paleo-Oxygen Levels and Diagenetic Zones. Journal of African Earth Science, 86, 25-44. https://doi.org/10.1016/j.jafrearsci.2013.05.002

Gibbs, R. J. (1970). Mechanisms Controlling World's Water Chemistry. Science, 170, 1088-1090. https://doi.org/10.1126/science.170.3962.1088

Hounslow, A. W. (1995). Water Quality Data: Analysis and Interpretation (pp. 397). New York: Lewis Publishers CRC Press.

ISO (2003). Standard ISO 5667-3: Water Quality-Sampling-Part 3: Guidance on the Preservation and Handling of Water Samples. Geneva: International Organization for Standardization.

ISO (2006). Standard ISO 5667-1: Water Quality-Sampling-Part 1: Guidance on the Design of Sampling Programs and Sampling Techniques. Geneva: International Organization for Standardization.

ISO (2009). Standard ISO 5667-11: Water Quality-Sampling-Part 11: Guidance on Sampling of Groundwaters. Geneva: International Organization for Standardization.

Kelley, W. P. (1940). Permissible Composition and Concentration of Irrigation Waters. Proceedings of the American Society of Civil Engineers, 66, 607-613. 
Kumar, K. S., Chandrasekar, N., Seralathan, P., Godson, P. S., \& Magesh, N. S. (2011). Hydrogeochemical Study of Shallow Carbonate Aquifers, Rameshwaram Island, India. Environmental Monitoring and Assessment, 184, 4127-4138.

Langguth, H. R. (1966). Groundwater Characteristics in Bereiech Des Velberter Sattles (pp. 127). North Rhine-Westphalia, Germany: Ministry of Agricultural and Land Management Research Düsseldorf.

Lloyd, J. A., \& Heathcote, J. A. (1985). Natural Inorganic Hydrochemistry in Relation to Groundwater: An Introduction (pp. 296). New York: Oxford University Press.

Lordon, A. E. D., Shandini, Y., Agyingi, C. M., Yossa, M. T., Stephane, K. T., \& Douglas, B. (2017). Structural Interpretation of the Mamfe Basin from Satellite Gravity Data (EGM 2008). Journal of Earth Sciences and Geotechnical Engineering, 7, 45-53.

Mamfe Council (2014). United Councils and Cities of Cameroon.

Ndip, A. E., Agyingyi, C. M., Nton, M. E., \& Oladunjoye, M. A. (2018). Review of the Geology of Mamfe Sedimentary Basin, SW Cameroon, Central Africa. Journal of Oil, Gas and Petrochemical Sciences, 1, 35-40. https://doi.org/10.30881/jogps.00008

Ndougsa-Mbarga, T., Manguelle-Dicoum, E., Campos-Enriquez, J. O., \& Atangana, Q. Y. (2007). Gravity Anomalies, Sub-Surface Structure and Oil and Gas Migration in the Mamfe, Cameroon-Nigeria, Sedimentary Basin. Geofísica Internacional, 46, 129-139.

Nguimbous-Kouoh, J. J., Takougam, E. M. T., Nouayou, R., Tabod, C. T., \& Manguelle-Dicoum, E. (2012). Structural Interpretation of the Mamfe Sedimentary Basin of Southwestern Cameroon along the Manyu River Using Audiomagnetotellurics Survey. Geophysics, 2012, Article ID: 413042. https://doi.org/10.5402/2012/413042

Nickson, R. T., McArthur, J. M., Shrestha, B., Kyaw-Nyint, T. O., \& Lowrt, D. (2005). Arsenic and Other Drinking Water Quality Issues, Muzaffargarh District, Pakistan. Applied Geochemistry, 20, 55-68. https://doi.org/10.1016/j.apgeochem.2004.06.004

Paliwal, K. V. (1972). Irrigation with Saline Water (pp. 198). New Delhi: Water Technology Centre, Indian Agricultural Research Institute.

Pandian, K., \& Sankar, K. (2007). Hydrochemistry and Groundwater Quality in the Vaippar River Basin, Tamil Nadu. Journal of the Geological Society of India, 69, 970-982.

Piper, A. M. (1944). A Graphic Procedure in the Geochemical Interpretation of Water Analysis. Eos, Transactions American Geophysical Union, 25, 914-928. https://doi.org/10.1029/TR025i006p00914

Richards, L. A. (1954). Diagnosis and Improvement of Saline Alkali Soils. Agriculture Handbook No. 60. Washington DC: US Department of Agriculture.

Sawyer, G. N., \& McCarthy, D. L. (1967). Chemistry of Sanitary Engineers (2nd ed.). New York: McGraw Hill.

Semiromi, F. B., Hassani, A. H., Torabian, A., Karbassi, A. R., \& Lotfi, F. H. (2011). Evolution of a New Surface Water Quality Index for Karoon Catchment in Iran. Journal of Water Science and Technology, 64, 2483-2491. https://doi.org/10.2166/wst.2011.780

Sisodia, R., \& Moundiotiya, C. (2006). Assessment of the Water Quality Index of Wetland Kalakho Lake, Rajasthan, India. Journal of Environmental Hydrology, 14, 1-11.

Todd, D. K. (1980). Ground Water Hydrogeology. New York: John Wiley and Sons, Inc.

Walton, W. C. (1970). Groundwater Resources Evaluation. New York: McGraw Hill Book Company.

Wilcox, L. V. (1995). Classification and Use of Irrigation Waters (pp. 19). Circular No. 
960, Washington DC: US Department of Agriculture.

World Health Organization (2017). Guidelines for Drinking-Water Quality: 4th Edition, Incorporating the 1st Addendum. Geneva: World Health Organization. 